\title{
fondation des centrales nucléaires
}

\section{french nuclear power plants foundation}

\author{
J.L. COSTAZ, M. HAGHGOU \\ E.D.F., S.E.P.T.E.N.*
}

\section{Résumé}

\begin{abstract}
Après une description rapide des études menées par E.D.F. pour le choix des sites et les caractéristiques géotechniques des sols, les problèmes d'interaction sol-structures dans le domaine statique sont exposés dans leur généralité et à l'aide d'exemples précis.

Enfin, les études de développement liées au retour d'expérience donnent des orientations pour l'avenir.
\end{abstract}

\section{Abstract}

After a rapid description of the studies made by E.D.F. for the choice of the nuclear sites and for determining the geotechnic data, the soil-structure interaction problems for static loadings are presented in a general way and accompanied by local examples.

Finally, development works related to the feedback experience provide an approach for the future. 


\section{INTRODUCTION}

En 1973, la France a décidé de lancer un programme d'équipement électronucléaire de grande ampleur devant permettre dix ans plus tard la fin de l'utilisation du pétrole pour la production d'électricité.

Ce programme a fait l'objet de deux paliers techniques caractérisés par la puissance électrique des unités :

34 tranches de $900 \mathrm{MWe}$ et 20 tranches de 1300 MWe ont été implantées sur une vingtaine de sites (figure 1). Comme ces sites sont répartis sur l'ensemble du territoire à l'exception des zones montagneuses, leur géologie fournit des conditions de fondations très différentes allant de vases de la Gironde (Blayais) au granite du Cotentin (Flamanville) en passant par les couches alluvionnaires des principaux fleuves français.

Les études concernant les fondations des centrales nucléaires ont fortement enrichi l'expérience d'E.D.F. et de ses ingénieurs-conseils : MECASOL, SIMECSOL, COYNE et BELLIER.

En effet, le fait de disposer d'ouvrages identiques sur des sols différents est une source d'information unique que nous continuons à exploiter pendant de nombreuses années après la construction par le suivi des tassements dans le temps.

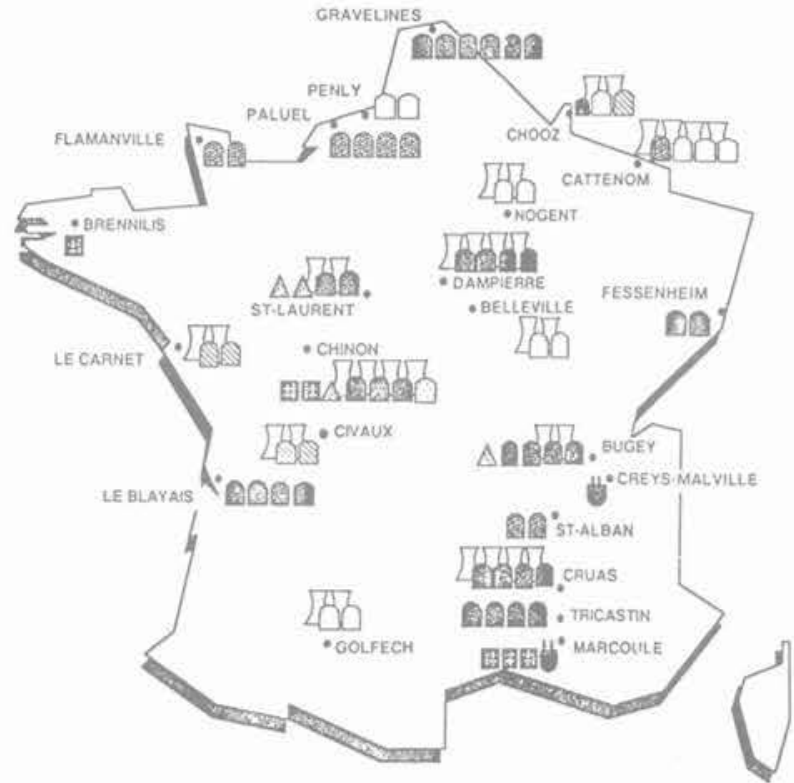

\begin{tabular}{|c|c|c|c|c|}
\hline TRANCHES & $\begin{array}{c}900 \mathrm{MW} \\
\text { REP }\end{array}$ & $\begin{array}{c}1300 \mathrm{MW} \\
\text { REP }\end{array}$ & UNGG & RNA \\
\hline en exploitation & (1) & 图 & $\Delta$ & 曲 \\
\hline en construction & 0 & D & & \\
\hline en projet & & Q & & \\
\hline déclassées & \multicolumn{4}{|c|}{ 田 } \\
\hline 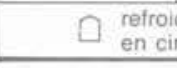 & $\begin{array}{l}\text { dissement } \\
\text { cuit ouvert }\end{array}$ & & refroi & $\begin{array}{l}\text { sement } \\
\text { it ferme }\end{array}$ \\
\hline
\end{tabular}

Fig. 1. - Les centrales nucléaires de la France. Fig. 1. - Location of french nuclear power plants

\section{DIVERS BATIMENTS D'UNE CENTRALE NUCLÉAIRE A EAU SOUS PRESSION}

\subsection{Description sommaire}

Une centrale nucléaire comprend plusieurs bâtiments indépendants, les divers radiers n'étant liés entre eux que dans des cas très particuliers.

C'est la solution la plus économique mais elle peut conduire à des tassements différentiels entre bâtiments qui doivent être pris en compte.

Le bâtiment le plus lourd est le bâtiment du réacteur de forme cylindrique. La charge au sol est en moyenne de $0.5 \mathrm{MPa}$. Il est entouré de bâtiments de forme parallélépipédique dont la charge au sol est d'environ $0,25 \mathrm{MPa}$. On distingue :

- le bâtiment du combustible (BK):

- le bâtiment des auxiliaires nucléaires (BAN);

- le bâtiment électrique et des auxiliaires de sauvegarde (BAS - BL) ;

- le bâtiment de liaison.

Enfin, la salle des machines est constituée de poutres et de poteaux fondés généralement sur semelles ( $\mathrm{SdM}$ ).

La figure $2 \mathrm{a}$ montre une vue générale de lî̀lot nucléaire pour le palier 1300 (P'4) et la figure $2 b$ une coupe passant par les bâtiments BK et BAS - BL.

La figure G1 (annexe 3) donne le plan de masse d'une centrale de 900 MWe à 2 tranches jumelées.

\subsection{Bâtiment du réacteur}

Le bâtiment du réacteur est fondé sur radier et comporte une enceinte en béton précontraint capable d'assurer le confinement des produits radioactifs libérés et la protection vis-à-vis du rayonnement émis par ces produits en cas d'incident et tout particulièrement en cas d'accident de perte de réfrigérant primaire. Elle assure également la protection contre les agressions externes.

Cette enceinte est appelée "enceinte de confinement ». Elle peut être constituée :

- soit par une enceinte simple en béton précontraint munie intérieurement d'une peau métallique d'étanchéité (tranches REP 900 MWe)

- soit par une enceinte à double paroi : enceinte interne en béton précontraint sans peau métallique d'étanchéité et enceinte externe en béton armé, ces parois étant séparées par un espace entre enceintes permettant la récupération et la filtration des fuites en provenance de l'enceinte interne (tranches REP $1300 \mathrm{MWe}$ ).

Comme indiqué sur la figure 3 , on distingue trois standards pour le palier REP 1300 :

- P4 dont la tête de série est PALUEL: 


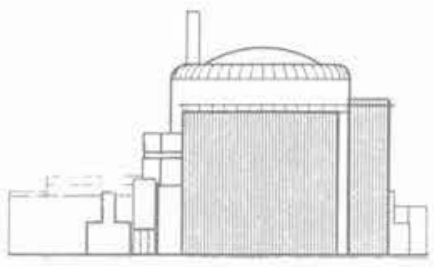

Vue suivant $A$

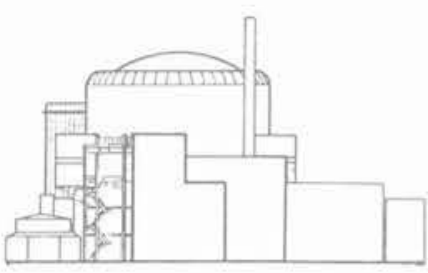

Vue suivant $\mathrm{C}$

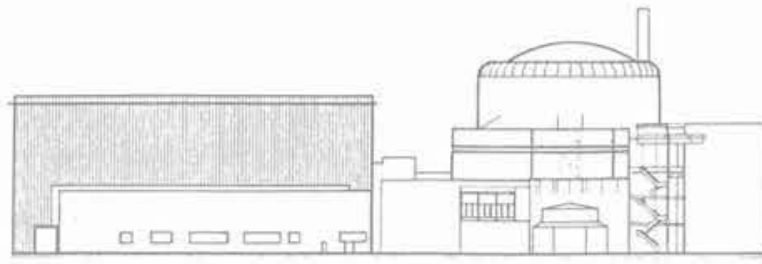

Vue suivant $B$

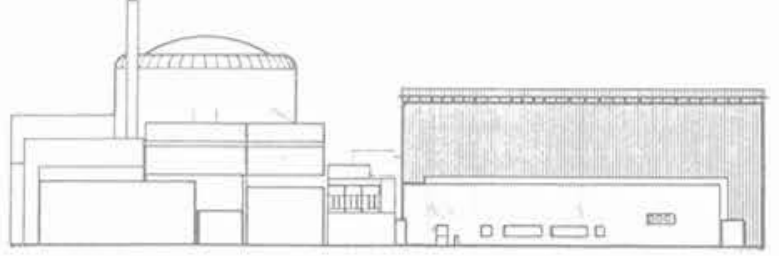

Vue suivant $D$

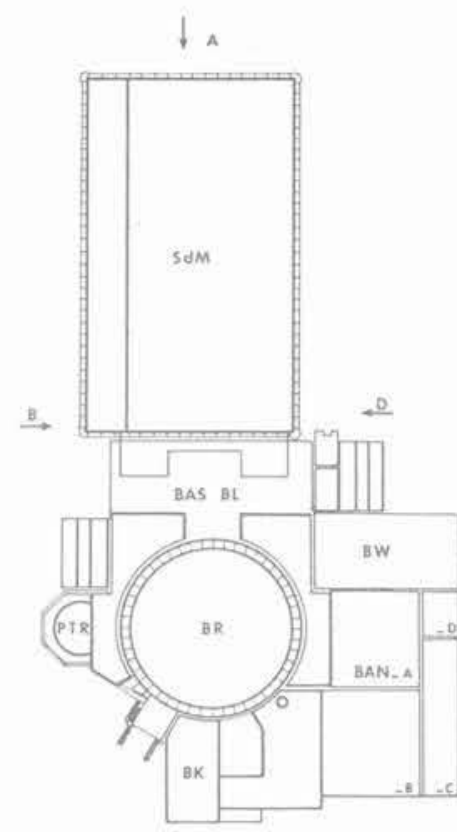

†c

Fig. 2.a - Vue générale du bloc usine, PWR 1300 P'4.

Fig. 2.a - General view of nuclear island, PWR 1300 P'4.

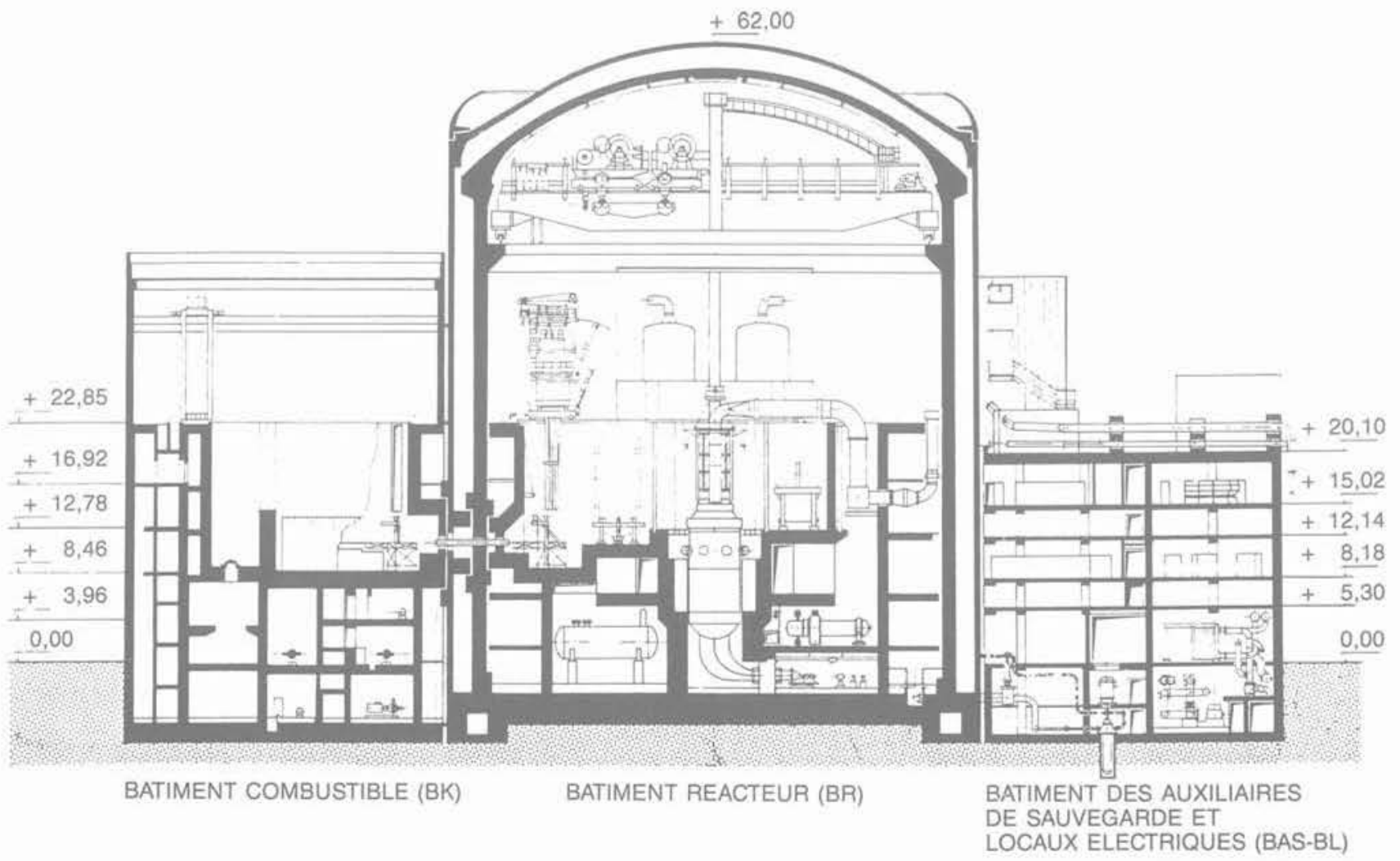

Fig. 2.b - Coupe AA - ilot nucléaire, PWR 1300 P'4.

Fig. 2.b - Cross section AA - nuclear island, PWR 1300 P'4. 


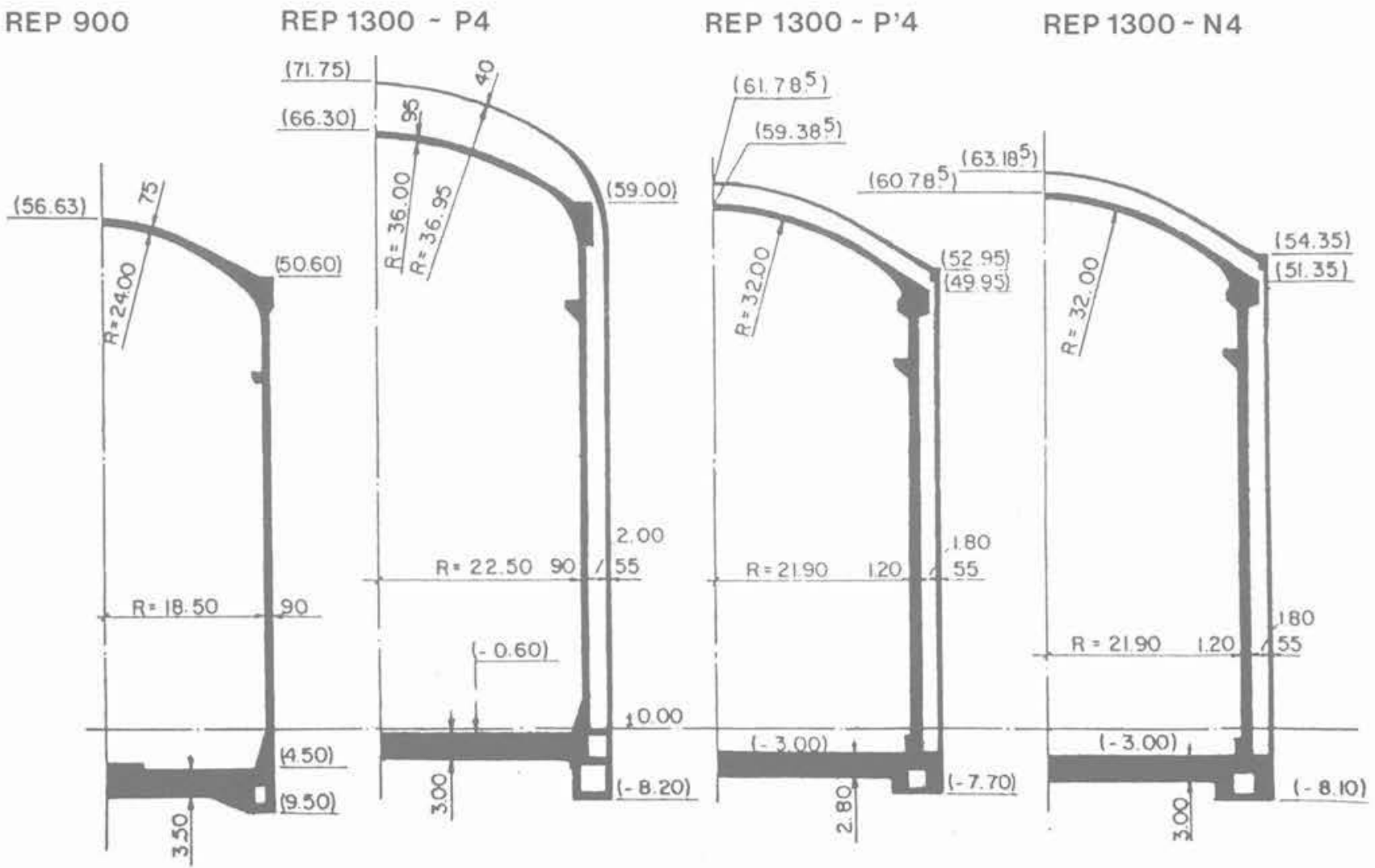

Fig. 3. - Différents standards d'enceintes de confinement.

Fig. 3. - French PWR containement vesse/s standards.

- P'4 dont la tête de série est CATTENOM:

- N4 dont la tête de série est $\mathrm{CHOOZ}$.

\section{Représentation de la structure}

Ce bâtiment est amené à subir des chargements variés au cours de son exploitation (poids propre, épreuve...) et il peut être amené à rencontrer des situations accidentelles. La nature et la distribution des efforts apportés en sous-face de son radier par le sol influencent très notablement son dimensionnement, notamment celui de sa partie inférieure.

En raison de la diversité des sols rencontrés sur les différents sites, et de la sensibilité des structures du bâtiment réacteur aux efforts apportés par le sol, la conception de ces ouvrages a toujours été orientée vers la définition d'une part de parties reconductibles sur tous sites et d'autre part de zones de l'ouvrage qu'il était nécessaire d'adapter à la nature des terrains rencontrés sur chaque site. Les parties non reconductibles sont généralement :

- le radier:

- les parties inférieures des structures internes lorsque celles-ci sont liées rigidement au radier:

- la partie inférieure (gousset de raccord avec le radier) des enceintes.
Il faut noter que les parties reconductibles ne sont pas toutes absolument insensibles à la nature du sol. Les parties basses des enceintes interne et externe, au-dessus des goussets sont nécessairement influencées, même faiblement, par les réactions du sol sous le radier. En conséquence, afin de pouvoir définir un dimensionnement standard de ces parties, des calculs enveloppes ont été faits, en prenant en compte plusieurs types de sols mou, moyen ou dur selon les dénominations habituelles.

\section{CHOIX DES SITES}

Avant de construire une centrale nucléaire, il faut trouver des sites convenables. Ceci se prépare longtemps à l'avance et fait l'objet d'une très large concertation.

Le choix d'un site de centrale nucléaire résulte de très nombreuses études prenant principalement en compte :

- les besoins de la région en énergie électrique;

- le réseau de transport;

- la proximité d'une source froide (mer ou rivière) nécessaire pour le refroidissement du condenseur de la turbine : 
- la direction des vents dominants;

- la densité de la population dans les environs du site ;

- l'impact visuel de la centrale dans l'environnement:

- la géologie du site;

- le risque sismique.

La nature et la qualité du sol de fondation sont prises en compte, mais ne sont pas des facteurs déterminants. Par contre, ces paramètres ont des conséquences importantes sur la construction de la centrale.

\section{4. ÉTUDE DU SOL D’UN SITE}

L'étude du sol d'un site rassemble des données précises dans les domaines suivants:

- la géologie qui décrit la nature et la disposition des terrains rencontrés sur le site à l'échelle géologique; elle inclut l'hydrogéologie qui étudie la pénétration et l'accumulation des eaux superficielles dans les terrains, l'importance et la position des réserves ainsi constituées et l'écoulement de la nappe phréatique ;

- lanalyse du risque sismique;

- la géotechnique qui détermine les caractéristiques mécaniques des couches de sol.

\subsection{La géologie et l'hydrogéologie}

L'étude géologique est à la base de toutes les études présentées ci-dessous. Elle est conduite d'abord à l'échelle régionale, puis à l'échelle locale.

Il est arrivé que cette étude aboutisse à la découverte d'une anomalie entraînant l'abandon du site. Par exemple le site de Fumay, où la présence d'une faille était soupçonnée, a été délaissé au profit de celui de Chooz.

L'hydrogéologie du site est soigneusement étudiée avant et après travaux. En effet, les travaux de terrassements peuvent modifier l'écoulement de la nappe phréatique, entrainant par exemple la diminution du débit d'exhaure des puits privés ou industriels de la région. Les travaux peuvent également mettre en communication la nappe phréatique avec une rivière polluée, entraînant une modification de ses caractéristiques chimiques.

En fin de travaux, E.D.F. établit une carte où figure l'écoulement de la nappe superficielle et, de plus, calcule le temps de transfert de la nappe de la centrale à l'exutoire le plus proche (rivière pour un site fluvial).

$\mathrm{Au}$ cours de la vie de la centrale, l'hydrogéologue effectue des contrôles périodiques de la nappe phréatique pour vérifier l'absence de pollution et la nonaltération de l'eau.

\subsection{L'analyse du risque sismique}

La protection des centrales nucléaires vis-à-vis des effets des séismes est un aspect important de la sûreté; elle passe par les trois étapes suivantes :

- la détermination des mouvements sismiques de référence;

- le dimensionnement des ouvrages;

- la mise en place d'une instrumentation de détection sismique sur le site.

\subsection{La géotechnique}

Les bâtiments des îlots nucléaires sont des ouvrages lourds qui appliquent au sol des contraintes comprises entre $0,25 \mathrm{MPa}$ et $0,5 \mathrm{MPa}$. Les services topographiques d'E.D.F. qui auscultent ces bâtiments au cours de leur construction, puis au cours de leur exploitation, mesurent des tassements moyens qui peuvent aller jusqu'à $30 \mathrm{~cm}$ et des tassements différentiels entre bâtiments de plusieurs centimètres qui s'accroissent pendant toute la vie de la centrale lorsque le sol est argileux.

Les déplacements différentiels entre bâtiments peuvent atteindre les limites prévues pour les matériels communs à ces bâtiments et principalement les tuyauteries. De plus, les hétérogénéités du sol de fondation entraînent un basculement des bâtiments qui peut gêner le fonctionnement des matériels.

Pour limiter les tassements des bâtiments, plusieurs techniques ont été adoptées quand le sol était trop mauvais :

- à Gravelines, réalisation sous l'îlot nucléaire d'un radier général de trois mètres d'épaisseur moyenne, constitué d'un sol ciment armé de deux nappes d'aciers :

- au Blayais, substitution de $15 \mathrm{~m}$ de vase de la Gironde par un remblai sableux mis en place par une technique hydraulique et construction de la prise d'eau sur pieux;

- à Belleville, consolidation de $10 \mathrm{~m}$ d'alluvions sablo-limoneuses de Loire par vibro-flottation;

- à Nogent, préchargement du sol de fondation des réfrigérants atmosphériques.

Ces techniques sont efficaces, mais onéreuses et, de plus, elles ne peuvent être généralisées.

Une solution élégante pour le spécialiste en génie civil est de demander au mécanicien d'augmenter les tolérances admises sur son matériel. Mais on comprend facilement que cette méthode a ses limites. Il faut donc essayer d'améliorer les connaissances en mécanique des sols et d'assurer avec les laboratoires de recherche et les ingénieurs-conseils des études pour perfectionner :

- les essais au laboratoire et in situ;

- les lois de comportement des sols destinées à simuler convenablement sur ordinateur les différents essais; 
- les calculs de prévision de tassement.

C'est le programme de travail que nous poursuivons depuis plusieurs années. Nous y reviendrons dans cet exposé.

\section{INTERACTION SOL-STRUCTURE}

Dans une étude d'interaction sol-structure, il intervient évidemment deux ensembles très différents qui sont le sol de fondation et la structure elle-même. L'interface des deux ensembles est nette et bien déterminée lorsqu'il s'agit de la fondation d'une centrale nucléaire. Les caractéristiques de la structure sont relativement bien connues, comparées à celles du sol.

Une analyse d'interaction sol-structure peut avoir deux objectifs distincts :

- la détermination de la déformée des radiers et l'amplitude des déformations (tassements absolus et différentiels) ;

- l'évaluation de la distribution des contraintes à l'interface, ainsi que les contraintes qui seront développées au sein de la structure.

Ces deux objectifs peuvent être atteints simultanément par une analyse globale, modélisation par éléments finis de l'ensemble de la structure et du sol sous-jacent. Mais, pour des raisons qui vont être développées plus loin, ce type d'analyse est réservé au bâtiment du réacteur. Pour les autres bâtiments, lanalyse est basée sur les résultats de calculs préalables pour lesquels il est d'usage de ne représenter la structure que par une schématisation de sa fondation. Parfois même, la structure est remplacée par une distribution de charge en surface du sol, déduite en admettant des hypothèses sur la rigidité de la fondation. Il se posera alors le problème délicat de la continuité des déplacements à l'interface sol-structure.

Dans l'ensemble. on peut prétendre que c'est la détermination des contraintes et des déformations du massif de sol qui restera la partie délicate de l'ensemble de l'étude.

\subsection{Calcul des tassements}

L'estimation de l'interaction sol-structure et l'évaluation des tassements à court et à long terme des îlots nucléaires, comme tout autre calcul de mécanique des sols, consiste à idéaliser le phénomène, donc à utiliser un "modèle mathématique» après avoir effectué une chaîne d'opérations destinée à fournir les données nécessaires à l'exécution du calcul. Pour réussir une bonne prévision, il faut réunir les facteurs suivants :

a) connaissance de la géométrie précise des couches de sol, et des conditions de drainage; b) bonne connaissance des caractéristiques in situ des matériaux, et de l'état de contrainte initial;

c) possession d'une modélisation adéquate pouvant représenter fidèlement la géométrie du problème, le comportement des sols et l'interaction sol-structures:

d) correspondance entre le planning de chargement original et le planning suivi sur le chantier. Dans le cas contraire, il est nécessaire de réviser le calcul en tenant compte de l'histoire réelle du chargement.

De plus, on doit pouvoir déterminer avec suffisamment de précision les paramètres à introduire dans le modèle. La défaillance d'un de ces facteurs suffit à détériorer les prévisions. Or, on comprend qu'il soit difficile de réunir toutes ces conditions lorsque l'on connait les contraintes imposées sur chaque site :

- les campagnes de prospection doivent être menées rapidement, leur budget est forcément limité. De ce fait, la gêométrie et les caractéristiques des couches de sol ne sont pas généralement connues avec beaucoup de précision ;

- les essais au laboratoire peuvent ne pas être parfaitement représentatifs du comportement du sol in situ et leurs résultats sont souvent dispersés. La cause doit en être cherchée dans l'hétérogénéité naturelle des sols, le remaniement des échantillons. l'effet de l'échelle et du temps.

De plus, il est difficile de connaître exactement les conditions de drainage des différentes couches du sol et le planning de construction est rarement connu avec précision lors des études de sol.

Au cours de la dernière décennie, des progrès considérables ont été effectués dans la mise au point des outils de calcul sophistiqués. Actuellement, l'ingénieur dispose de codes de calcul aux éléments finis tridimensionnels incorporant des lois rhéologiques complexes, sensés bien représenter le comportement réel du sol, ainsi que l'interaction sol-structure. Mais ces codes sont lourds d'utilisation, et donc très onéreux; de plus, la détermination des paramètres des lois rhéologiques complexes pose encore beaucoup de problèmes. C'est pourquoi les modélisations usuelles, dans la pratique industrielle, sont encore basées sur des simplifications successives de la réalité. En effet, l'expérience montre que des discordances entre les calculs prévisionnels et des mesures effectuées sur les ouvrages ont souvent pour cause une mauvaise détermination de la géométrie et des caractéristiques in situ des couches du sol.

Le sol est un milieu discontinu, anisotrope, hétérogène avec un comportement non linéaire. Dans les méthodes usuelles de calcul de tassement, on adopte des simplifications. C'est au niveau du choix de ces simplifications que les méthodes utilisées par différents bureaux d'études se distinguent. Certains, s'inspirant de la loi hyperbolique de DUNCAN, tendent à avantager le comportement non linéaire du sol, tandis que. d'autres adoptent un comportement linéaire pour le sol, mais tiennent compte dans une certaine mesure de l'influence de l'anisotropie et de l'hétérogénéité verticale des couches du sol pour le calcul des contraintes. 


\section{2. - Cas du bâtiment du réacteur}

Pour tout nouveau site, et parfois même à l'intérieur même d'un site pour les différentes tranches si des hétérogénéités des sols le justifient, le bâtiment réacteur des centrales nucléaires fait l'objet d'un calcul d'ensemble aux éléments finis (en général axisymétrique pour le modèle, mais susceptible de prendre en compte des chargements non axisymétriques). Les éléments de structure représentés sont :

- les enceintes interne et externe, avec leur dôme;

- le radier:

- les structures internes du bâtiment réacteur, sous une forme simplifiée toutefois.

La représentation du sol est obtenue de deux façons. La première utilise des éléments finis (volumiques). On modélise alors les couches de sol, avec leurs différents modules jusqu'à une distance de l'ordre de deux fois le rayon du bâtiment en direction radiale, et une fois et demie en profondeur (modèle "bac de sols, figure 4).

Le principal inconvénient de semblables modèles est bien évidemment la taille des calculs qu'ils engendrent compte tenu de la multiplicité des cas de charge pour une précision parfois illusoire par suite des incertitudes sur les caractéristiques des sols à moins de réaliser plusieurs modèles pour tester la sensibilité.

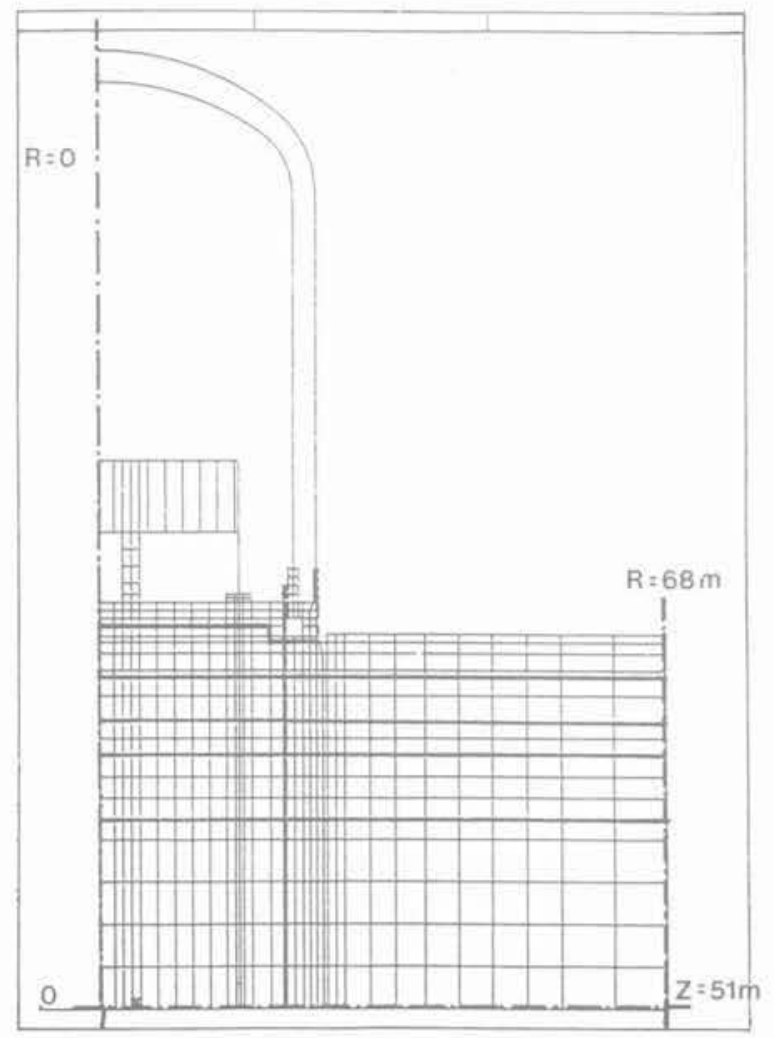

La deuxième solution, plus économique en temps de calcul, utilise une modélisation du sol par des ressorts (coefficient de réaction). Si elle conduit à des modèles de taille plus réduite, cette méthode pose toutefois le problème de la définition des raideurs des ressorts. Ces dernières sont en général obtenues en cherchant à retrouver sous le bâtiment réacteur les tassements prévus par les calculs géotechniques. Afin d'avoir des hypothèses encadrantes en ce qui concerne la distribution des contraintes sous le radier, deux modélisations sont le plus souvent adoptées :

- une avec des ressorts de raideur uniforme sous tout le radier:

- une autre comportant deux zones de raideurs différentes, les plus grandes raideurs étant prévues en périphérie du bâtiment sous les enceintes, ce qui permet de retrouver une distribution de contraintes présentant des valeurs plus élevées au bord analogues à celle que donne un modèle "bac de sol " (modèle "ressorts", figure 4).

Dans la pratique, les deux méthodes sont utilisées. Pour le poids propre, il est vérifié que les deux modèles "ressorts" encadrent bien les efforts et déformations calculés par un modèle "bac de sol». Les modèles "ressorts" ainsi testés sont alors utilisés pour tous les autres cas de charge et notamment les cas sismiques avec prise en compte du décollement éventuel d'une partie du radier.
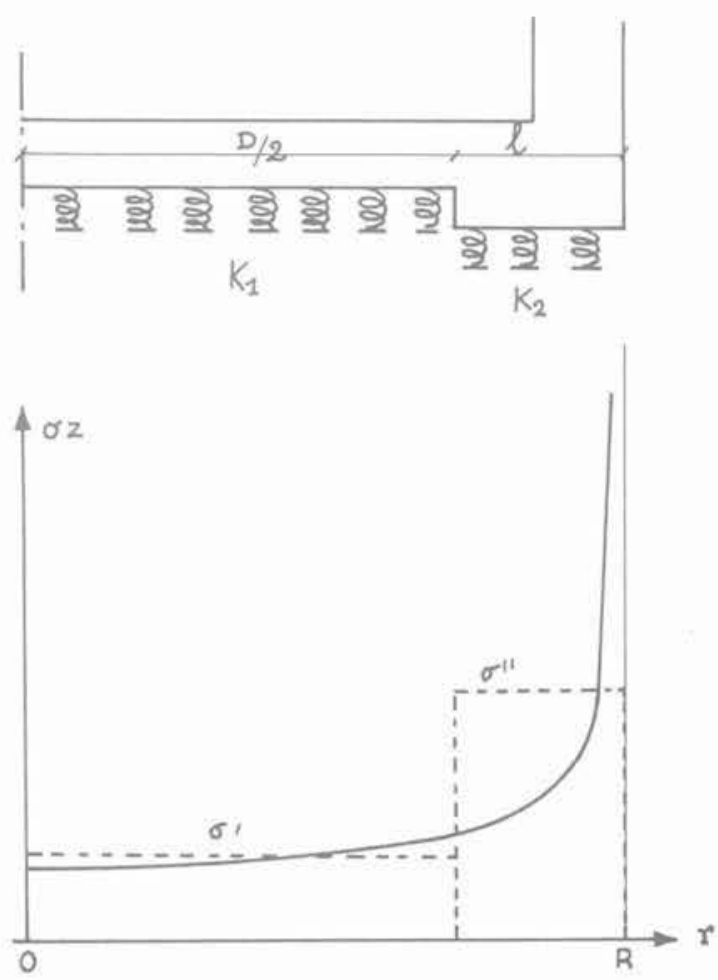

Fig. 4. - a) Exemple de modèle de calcul du bâtiment réacteur sur "bac de sol». b) Représentation du sol par des ressorts de raideur variable.

Fig. 4. - a) Global finite elements representation of soil-structure. b) Method of substructure with variable spring rigidity. 
Les différents types de chargements auxquels est soumis le bâtiment réacteur imposent de modéliser des caractéristiques statiques (construction, service normal, etc.), statiques rapides (épreuve) ou dynamiques (séisme, accidents, etc.). Différents modèles aux éléments finis sont donc réalisés, que l'on soumet ensuite aux chargements qui leur sont propres. Les résultats des efforts sont alors cumulés pour le dimensionnement.

Si comme nous venons de le voir, les problèmes liés à la nature du sol interviennent de façon importante au stade des calculs de dimensionnement, ils ont aussi une grande influence sur la conception même de l'ouvrage, notamment pour le radier et les galeries situées sous le radier.

\section{DISPOSITIFS D'AUSCULTATION}

La sensibilité des installations des bâtiments nucléaires aux tassements différentiels a fait sentir très tôt le besoin d'un contrôle continu du comportement réel de ces bâtiments au cours et après leur construction. La nature, le nombre et l'implantation des dispositifs de mesure peut différer d'un site à un autre, suivant le fait que le site est jugé sensible ou non.

En ce qui concerne le bâtiment du réacteur, les dispositifs de mesure relatifs à l'étude de l'interaction solstructure comprennent :

- Les mesures des déplacements et déformations d'ensemble :

- mesures topographiques des déplacements du radier et de la structure;

- déformée du radier par pots de nivellement à niveau d'eau, noyés dans le béton:

- déformée de la paroi cylindrique verticale à l'aide de pendules verticaux:

- diamètre du radier avec deux extensomètres horizontaux.

- Les mesures locales dans le béton :

- contraintes à l'aide des témoins sonores noyés dans le béton;

- températures à l'aide de thermocouples noyés dans le béton;

- contraintes de la peau métallique d'étanchéité;

- tension des câbles de précontrainte verticaux à l'aide de dynamomètres.

- Les mesures dans le sous-sol de fondation :

- pression de sol sous le radier à l'aide de cellules GLÖTZL;

- pressions interstitielles en profondeur à l'aide de capsules TÉLÉMAC;

- tassement des couches profondes;
- mesure du niveau de la nappe phréatique.

Dans un premier temps, ces mesures étaient surtout destinées à la détection des anomalies éventuelles par rapport aux prévisions des calculs (et réajustement des calculs d'interaction sol-structure). Par la suite, elles ont constitué une base de données pour des comparaisons calcul-expérience permettant une meilleure compréhension des phénomènes et le comportement réel des ouvrages dans le but de l'amélioration des procédures de calcul. Elles peuvent servir aussi pour établir les bases d'une procédure pour la révision de l'évaluation des tassements à long terme en se basant sur des mesures au cours et après la construction de la centrale.

\section{RETOUR D'EXPÉRIENCE RECHERCHE ET DÉVELOPPEMENT}

E.D.F. dispose, pour conduire une étude sur les tassements, d'une richesse d'information unique : 20 sites ont été équipés de centrales standardisées à eau pressurisée; chaque bâtiment réacteur constitue une sorte d'« essai à la plaque» en vraie grandeur. Il y a donc là matière à une banque de données à laquelle il sera toujours possible de se référer dans l'avenir pour porter un jugement global sur tel ou tel type de modélisation.

En liaison avec ce retour d'expérience, nous avons engagé en 1985 une étude destinée à définir une méthodologie nouvelle dans les prévisions de tassements. La démarche est déterministe et cherche d'une part à valoriser au mieux les techniques d'essais et de calculs existants, d'autre part à mettre au point de nouvelles approches théoriques et expérimentales.

Traditionnellement, l'appareil de laboratoire à la base des calculs de tassements est l'œedomètre : l'échantillon de sol, haut de $2 \mathrm{~cm}$, est chargé par paliers pour déterminer l'indice de compressibilité du matériau.

In situ, le pressiomètre permet d'exercer des pressions croissantes sur la paroi d'un forage et de mesurer le module pressiométrique qui, introduit dans des formules empiriques, conduit également à une prévision des tassements.

Les deux méthodes, assez rudimentaires, aboutissent, notamment sur les sites E.D.F., à des prévisions souvent discordantes avec des écarts pouvant aller du simple au double, comme cela a été le cas à Golfech sur l'emplacement des futurs aéroréfrigérants, ou à Nogent-sur-Seine sous lîlot nucléaire.

La question posée est donc la suivante : comment relier les deux méthodes, critiquables sur bien des points, et aboutir à une prévision réaliste?

La réponse passe par la modélisation numérique du comportement du sol, à l'aide de lois élastoplastiques représentatives de la physique réelle du matériau. Le schéma suivant indique la nouvelle procédure mise en œuvre.

E.D.F. est en mesure d'utiliser comme loi de comportement du sol, la loi élastoplastique multimécanisme 


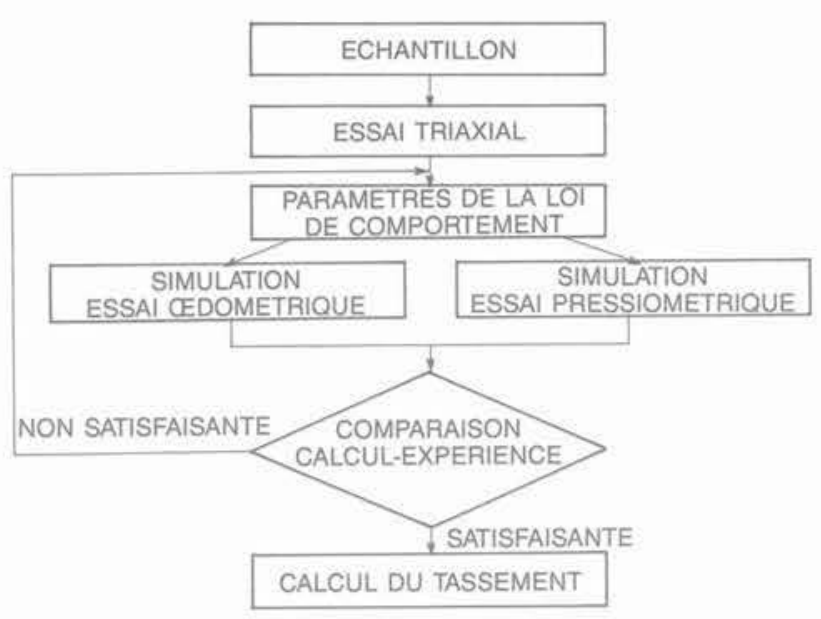

cyclique de HUJEUX (1985), développée par les équipes de chercheurs de l'École Centrale de Paris. Cette même loi sert à d'autres applications telles que les calculs de barrages en terre et, sous des formes plus ou moins modifiées, à l'extérieur d'E.D.F. (Institut Français du Pétrole, B.R.G.M., COYNE \& BELLIER) : elle devrait donc être amenée à connaître un grand développement.

La loi cyclique est introduite dans un code d'éléments finis tridimensionnel, qui se propose de traiter, non seulement les problèmes de tassements précédemment définis, mais encore les problèmes beaucoup plus lourds de calcul des contraintes et déformations dans le sol sous sollicitations dynamiques (séismes).

Parallèlement à ces applications théoriques, il convient de développer les outils expérimentaux qui soient en mesure d'accéder valablement aux paramètres du sol qui seront introduits dans les codes de calcul. Au laboratoire et in situ. l'effort de recherche d'E.D.F. est fourni en collaboration avec l'École Centrale. Pour l'expérimentation in situ, le pressiomètre autoforeur mis au point par le L.C.P.C." et le pressiomètre piloté par calculateur mis au point par la Société MENARD. sont testés, notamment dans la craie pâteuse du site de Nogent.

Pour terminer ce rapide tour d'horizon sur les calculs de tassements, précisons que les tassements à long terme, dont l'importance peut être capitale pour les liaisons entre bâtiments, font l'objet d'études très attentives visant d'une part à mettre au point les méthodes d'extrapolation à l'infini des valeurs de tassements observés pendant les premiers mois suivant la construction, d'autre part à introduire dans les codes de calcul existants des lois modélisant convenablement le fluage des sols.

Nous donnons en annexe quatre exemples de comparaison entre les calculs prévisionnels et les mesures réalisées in situ.

\section{RÉFÉRENCES}

BECUE P., JULIEN J.F., COUSIN M., DUPAS J.M., DORE M. (1981), Comportement du réacteur de Creys-Malville, 10th Int. Conf. Soil Mech. Found. Engng (I.C.S.M.F.E.), Stockholm, vol. 2, pp. 37-40.

BROWN P.T. (1969), Numerical Analyses of Uniformly Loaded Circular Rafts on Elastic Leavers of Finite Depth, Géotechnique, vol. 19, $\mathrm{n}^{\circ} 3$. pp. 399-404.

BURLAND J.B., BROMS B.B. and DE MELLO V.F.B. (1977), Behaviour of Foundations and structures. State of the Art Report, proc. 9th Int. Conf. Soil Mech. Found. Engng. (I.C.S.M.F.E.), Tokyo 1977. vol. 2, pp. 495-546.

COUSIN M., GNIEWEK J., JULLIEN J.F. (1978), Mesure de nivellement automatique, Vle Congrès inter. de l'analyse expérimentale des contraintes, V.D.1. Berichte NR 313, Munich, pp. 887-891.

DAVESNE M., BORDES J.L., BOUVARD A., DUPAS J.M., LEFEVRE A. (1983), Contrôle d'un préchargement de fondation à Nogent-sur-Seine, symp. Inter., essais en place, Paris 1983, vol. 2, pp. 19-24.

DOUSSOT M., DORE M., COLOMBET G. (1987), Comportement du réacteur de Creys-Malville vis-à-vis de la construction d'un bâtiment adjacent (A.P.E.C.), Colloque international-Interaction Sols-Structures, Paris, 5-7 mai 1987, pp. 39-46.

DUNCAN J.M. and CHANG C.Y. (1970). Nonlinear Analysis of stress and stain in soils, J. Soil Mech. \& Fnds. Div. proc. A.S.C.E., vol. 96. SM 5, pp. 1629 1653.

GIROUD J.P. (1972), Tables pour le calcul des fondations, Dunod-Paris.

HUJEUX J.C. (1985), Une loi de comportement pour le chargement cyclique des sols, Génie parasismique, Presses des Ponts-et-Chaussées, Paris, pp. 57-72.

MOREL A., ZOLZETTICH A., JULLIEN J.F. SOMMA E., AMIR-MAZAHERI D. (1979), Comparison between a $3 D$ photo-elastic model and a axisymetric finite element calculus, 5th Inter. Conf. Struc. Mech. in Reactor Techn. (S.M.I.R.T.), M 3.8.-1-8. Berlin-Ouest.

PALMER L.A. and BARBER E.S. (1940), Soil displacement under a circular loaded area, proc. High Res. Board, vol. 20, pp. 279-286; 319-332.

SIGISMOND J., DUPAS J.M., LEFEVRE A. (1983). La craie à Nogent-sur.Seine. Revue françaíse de Géotechnique, $\mathrm{n}^{\circ} 23$, pp. 5-17.

WORKING GROUP FROM E.D.F./SEPTEN (1983), The use of soil improvement technique in realisation of the French Nuclear Power programme, proc. of 8th Europ. Conf. Soil Mech. Found. Engng, Helsinki, May 1983, Communication 1.17.

\footnotetext{
- Laboratoire Central des Ponts-et-Chaussées.
} 


\section{ANNEXES - CONFRONTATION CALCUL - OBSERVATIONS}

\author{
Annexe 1 - Cas de la centrale \\ de Creys-Malville (RNR $1200 \mathrm{MWe}$ )
}

Le site de Creys-Malville est situé en rive gauche du Rhône, à $60 \mathrm{~km}$ en amont de Lyon. La centrale Super-Phénix appartient à la filière des réacteurs « surrégénérateurs », à neutrons rapides, refroidis au sodium, et délivre une puissance électrique de $1200 \mathrm{MWe}$.

Le bâtiment réacteur comporte une enceinte cylindrique en béton armé de $64 \mathrm{~m}$ de diamètre intérieur (épaisseur des murs $1 \mathrm{~m}$ ) et de $80 \mathrm{~m}$ de haut environ abritant les structures internes, avec un radier circulaire de $66 \mathrm{~m}$ de diamètre et de $5,3 \mathrm{~m}$ d'épaisseur fondé à $6 \mathrm{~m}$ environ de profondeur sous la plate-forme générale du site; le poids total du réacteur (radier compris) est de l'ordre de $1750 \mathrm{MN}$. Le réacteur est entouré de 7 bâtiments adjacents ( 4 générateurs de vapeur et 3 bâtiments des auxiliaires nucléaires) représentant $1175 \mathrm{MN}$. La salle des machines, située à proximité, représente un poids total d'environ $1300 \mathrm{MN}$ (figure C1).

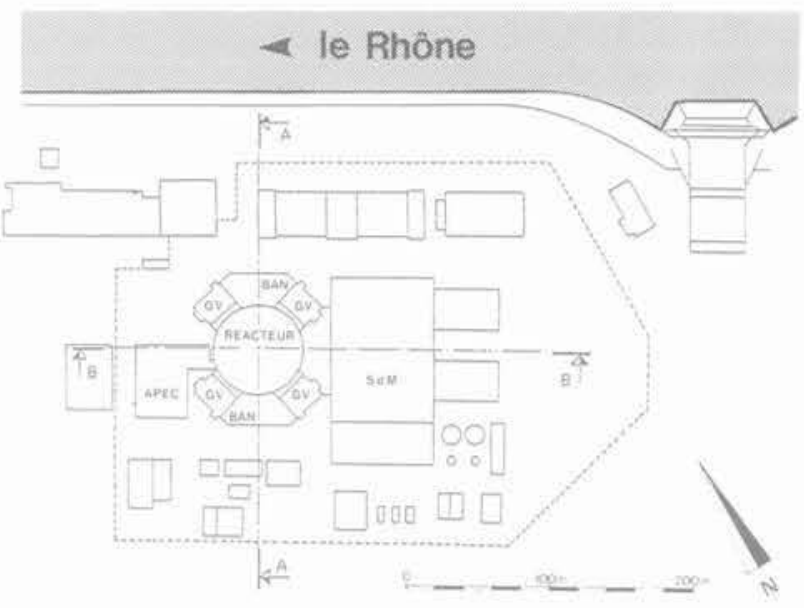

Fig. C1. - Centrale de Creys-Malville.

L'altitude du site est comprise entre $205 \mathrm{NGF}$ et 225 NGF (plaine alluviale au bord du fleuve et terrasse en contre-haut). Les ouvrages principaux sont implantés dans la plaine alluviale mais débordent légèrement sur la terrasse (TN : 205 NGF à 215 NGF). Les reconnaissances réalisées ont fait appel à des techniques variées (géophysique, sondages carottés, essais in situ et en laboratoire,...) et ont permis de définir la stratigraphie et les caractéristiques des matériaux. La stratigraphie est du haut vers le bas (figures $\mathrm{C} 2$ et $\mathrm{C} 3$ ) :

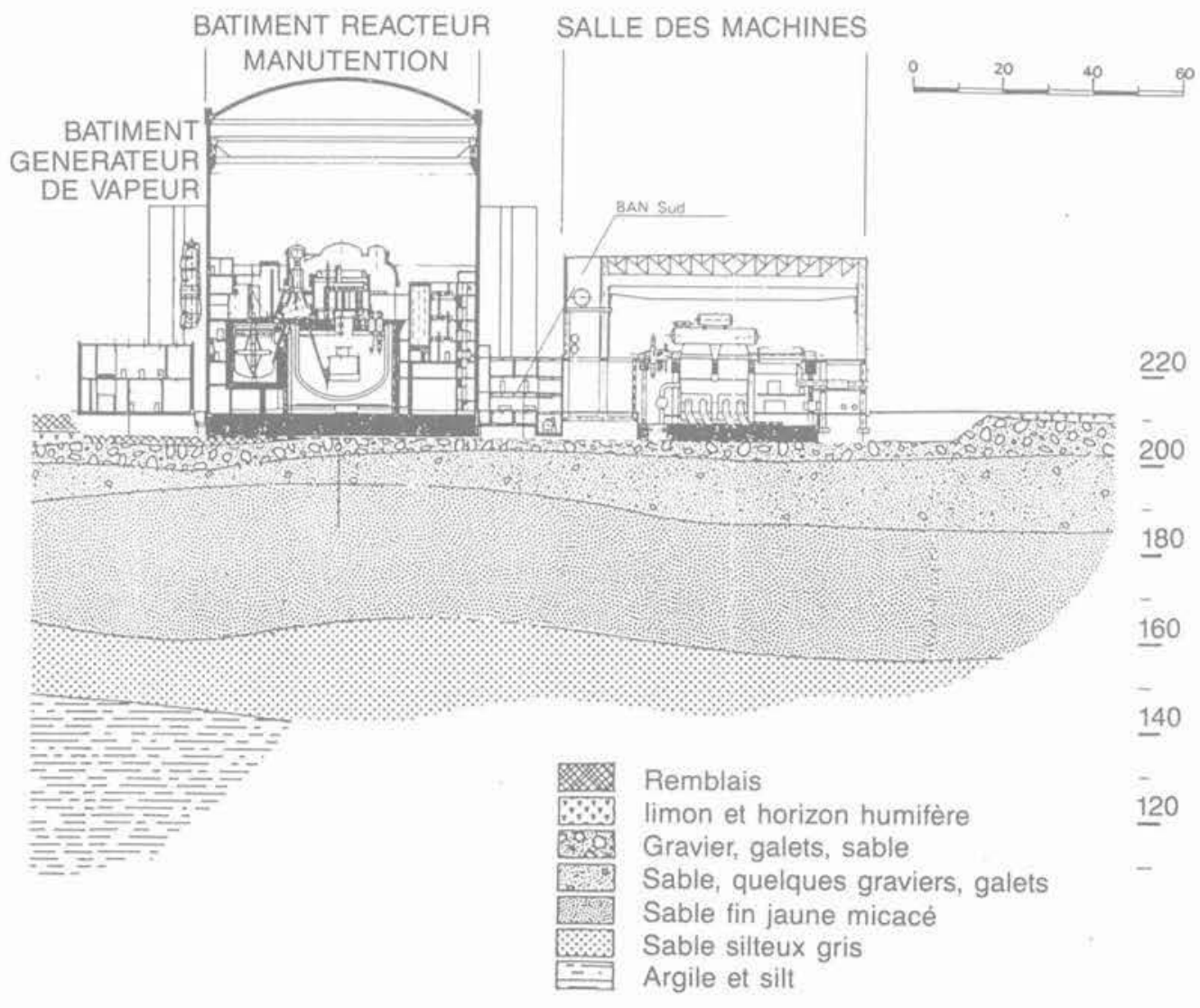

Fig. C2. - Creys-Malville - Coupe longitudinale.

Fig. C2. - Schematic cross section. 


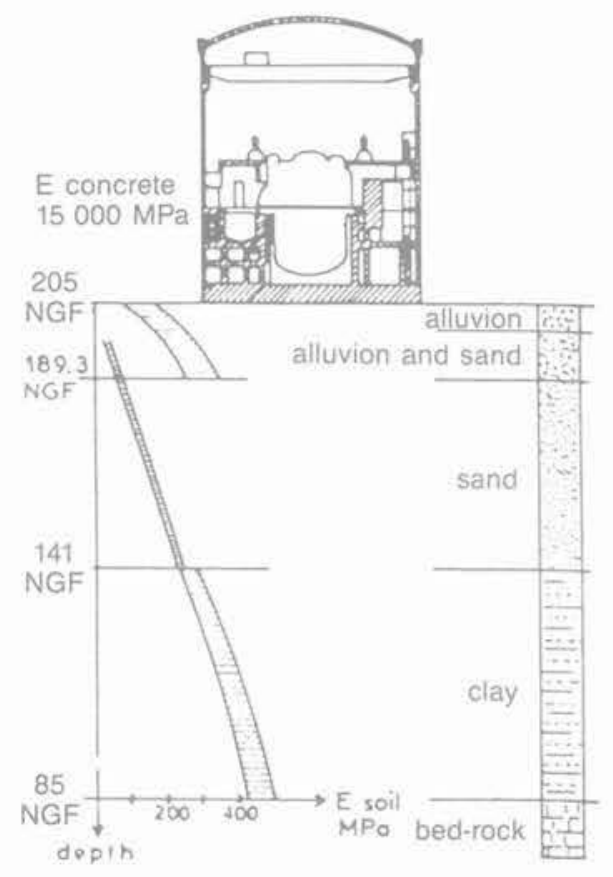

Fig. C3. - Coupe schématique du réacteur et des couches du sol.

Fig. C3. - Cross section of reactor building and soil characteristics

- limons de surfaces plus ou moins graveleux:

- alluvions sablo-graveleuses:

- sables moyens-fins à silteux:

- argile silteuse surconsolidée:

- substratum calcaire.

Les limons ont été décapés et remplacés par des alluvions sablo-graveleuses compactées pour constituer la plate-forme ou la fondation des ouvrages.

Les caractéristiques des sols ont été déterminées à partir d'essais in situ et en laboratoire sur échantillons intacts.

Les essais triaxiaux ont permis d'obtenir pour différents matériaux des lois du module de déformation $E$ en fonction de la contrainte effective moyenne et de la déformation axiale, avec une interprétation hyperbolique des courbes effort-déformation au voisinage de l'origine.

\section{Calculs prévisionnels}

Pour étudier l'interaction sol-radier conduisant au dimensionnement de la fondation et aux prévisions de tassement du bâtiment, deux approches ont été suivies. L'une visait à établir ces éléments à partir de calculs aux éléments finis modélisant le sol et la structure, et l'autre s'appuyait sur le comportement d'un modèle photoélastique reproduisant la géométrie du bâtiment et le sol de fondation (MOREL et al., 1979).
Le premier calcul aux éléments finis utilisait un modèle axisymétrique dans lequel le sol, jusque vers $110 \mathrm{~m}$ de profondeur, était divisé en 4 couches horizontales (figure C4).

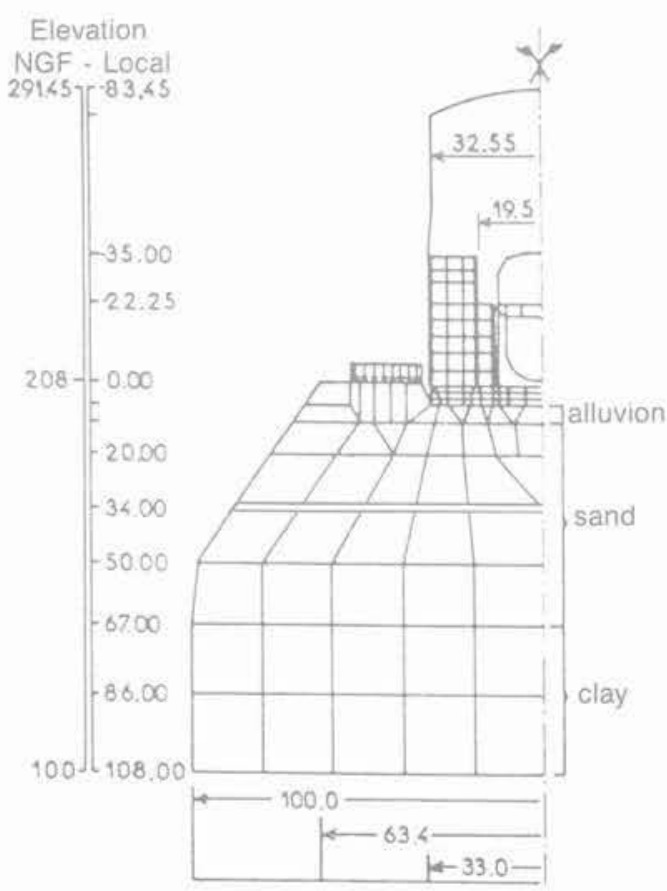

Fig. C4. - Modélisation axisymétrique. Fig. C4. - Axisymetric Model.

Le deuxième calcul aux éléments finis a été réalisé à 3 dimensions avec le programme ASKA. Il représente le sol (sur $120 \mathrm{~m}$ d'épaisseur) et la structure à l'aide d'un maillage tridimensionnel très élaboré permettant de tenir compte des dissymétries de charge (notamment de l'influence des bâtiments entourant le réacteur) ainsi que la variation en plan et en profondeur du module de déformation des sols. Les valeurs de $E$ sont déduites de calculs préliminaires, la répartition en profondeur des modules en tout point est calculée à partir de l'état des contraintes et des lois de module.

Les deux calculs aux éléments finis sont concordants pour le tassement au centre du radier (figure C5). Le second calcul fait apparaître une inclinaison du bâtiment réacteur suivant le diamètre NE-SO (l'extrémité $\mathrm{SO}$ tasse le plus). Les deux calculs conduisent à une flèche moyenne du radier d'environ $0,4 \mathrm{~cm}$. Le calcul tridimensionnel montre que la flèche maximale est obtenue sur le diamètre sensiblement E-O $(0.7 \mathrm{~cm})$.

Le modèle photoêlastique représentait la structure du bâtiment par une maquette au $1 / 200$ réalisée en résine époxy, tandis que la modélisation du sol était obtenue à l'aide de 29 disques en élastomère silicone de modules de déformation différents adaptés au profil du sol sur $120 \mathrm{~m}$ d'épaisseur. Les résultats obtenus sont en assez bonne concordance avec les calculs précédents (figure C5). 


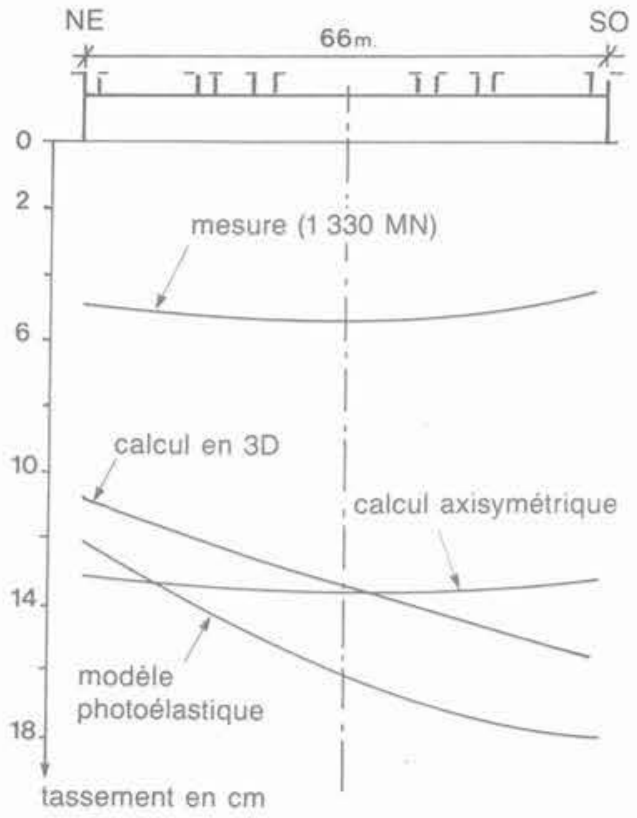

Fig. C5. - Déformée du radier suivant le diamètre NE-SO. Résultats des calculs et des mesures.

Fig. C5. - Deformed Base Mat - Calculated and Measured.

\section{Dispositifs d'auscultation}

Afin d'avoir les renseignements sur le comportement de l'inter-face sol-structure dès le début de la construction, des appareils de mesure ont été mis en place dans l'épaisseur du béton de propreté (figure C6) :

- un système de nivellement hydraulique automatique constitué de 19 pots de mesure;

- 17 cellules de contrainte du type GLÖTZL au contact avec le sol;

\section{- 5 thermosondes.}

Après construction du radier, la mise en place de 12 repères de nivellement à la surface de la fondation'a permis une surveillance topographique et la vérification des informations données par le système hydraulique (figure $\mathrm{C} 6$ ).

\section{Comportement du bâtiment réacteur}

Au cours de la construction du radier, la déformée observée en "bol " correspond à celle d'une surface de charge intermédiaire entre l'hypothèse souple (chargée uniformément) et l'hypothèse rigide (figure C7).

Dans l'ensemble, les observations concordent relativement bien avec les résultats des calculs prévisionnels. Ceci mis à part l'orientation du dévers (et en quelque sorte sa valeur à long terme).

Fin 1986, le tassement moyen du bâtiment réacteur était $10 \mathrm{~cm}$. Cette valeur est en bon accord avec les résultats de l'ensemble des calculs prévisionnels (12 à $14 \mathrm{~cm}$ de tassement final)

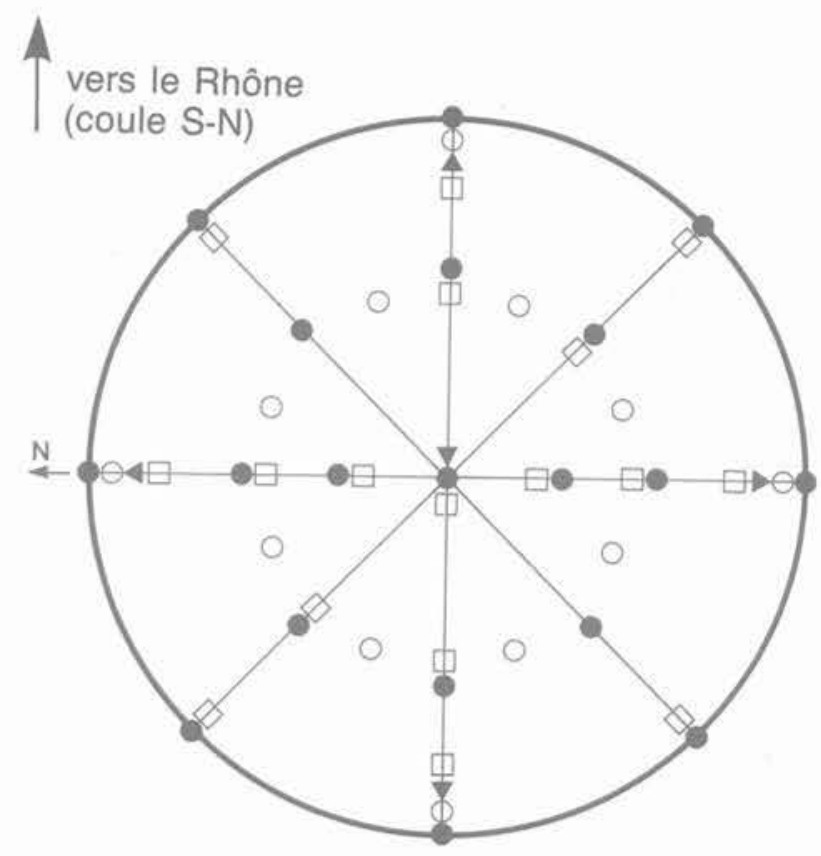

- Pot de nivellement

O- Plot topographique (surface du radier)

$\square$ - mesure de pression

A - mesure de température

Fig. C6. - Dispositif d'auscultation du radier. Fig. C6. - Measurement Devices on Base Mat.

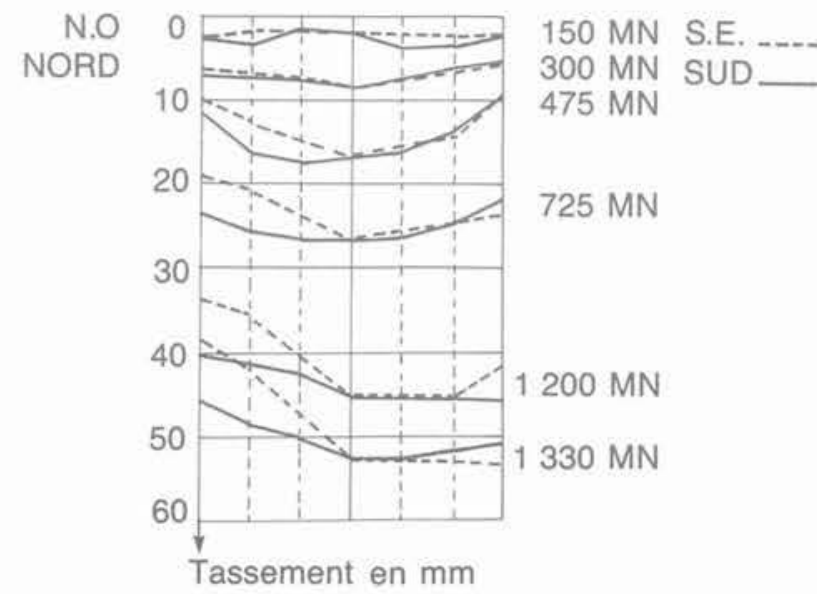

Fig. C7. - Évolution de la déformée du radier. Fig. C7. - Evolution of Base Mat Deformation. 
Quant au dévers final prévu, sa valeur variait de 2 à $9 \times 10^{-4}$ selon les calculs, et son orientation était prévue sud-ouest à ouest-sud ouest. Cette orientation découlait directement des hypothèses faites sur la géométrie des couches (sans prendre en compte le planning de chargement). Sur le site, l'importance et la direction du dévers ont beaucoup varié au cours de la construction. Cependant, depuis la relative stabilisation de la charge survenue fin 1980 , la direction du dévers s'est presque fixée vers le nord-est et sa valeur actuelle est de l'ordre de $6 \times 10^{-4}$.

On peut reconnaître que cette valeur $(6 \mathrm{~mm}$ sur $10 \mathrm{~m})$ paraît tout à fait normale pour un bâtiment de cette taille construit sur un sol hétérogène, et qu'il est très difficile de prévoir par le calcul avec une grande précision, le dévers de tels bâtiments. Mais, étant donné la conception particulière du réacteur Super-Phénix, la valeur admissible de dévers était fixée à $15 \times 10^{-4}$ (à compter du réglage de la cuve). De nouvelles études ont été alors entreprises pour retrouver par le calcul, l'évolution du dévers au cours du temps, ainsi que sa valeur et son orientation finale. Il s'est avéré qu'il aurait fallu retenir des hypothèses de calcul beaucoup plus détaillées, afin de prendre en compte les principaux facteurs susceptibles d'influencer le dévers. En particulier, l'influence des facteurs suivants a été réétudiée

- prise en compte de la géométrie plus précise des couches du sol;

- dissymétrie des terrassements généraux;

- consolidation de la couche argileuse:

- excentricité de la charge du bâtiment réacteur;

- planning précis de chargement.

DOUSSOT et al. (1987) traitent plus en détail ce sujet et, en particulier, l'influence de la construction tardive d'un des bâtiments annexes (A.P.E.C.) sur l'orientation et l'évolution du dévers du bâtiment réacteur.

\section{Annexe 2 - Cas du Blayais : Estuaire de la Gironde (REP 900 MWe)}

Au Blayais, la stratigraphie des terrains est la suivante :

- argile organique très compressible, sur près de $13,50 \mathrm{~m}$ d'épaisseur, ayant un angle de frottement interne nul, une cohésion de 4 à $35 \mathrm{KPa}$;

- alluvions sablo-graveleuses, de $5,50 \mathrm{~m}$ d'épaisseur moyenne et $35^{\circ}$ d'angle de frottement interne;

- en substratum, l'Eocène reconnu sur $140 \mathrm{~m}$ et composé :

- sur 15 m d'épaisseur, de marno-calcaire d'angle de frottement interne nul et de cohésion : $150 \mathrm{KPa}$ :

- de sables gris d'angle de frottement interne $36^{\circ}$.

Il a été décidé d'effectuer une substitution du terrain naturel jusqu'à la cote (- 13) NGF par du sable com- pacté. Après réalisation d'une paroi moulée, ancrée en tête, on a procédé au dragage, sous l'eau, du terrain compressible, et rabattu la nappe phréatique jusqu'à $8 \mathrm{~m}$ sous le fond de fouille, créant ainsi un gradient hydraulique agissant sur la partie supérieure de l'Eocène. Ceci a permis de compléter la stabilité de la paroi par des tirants inclinés et ancrés dans l'Eocène à l'arrière de celle-ci. Le sable fin de remblai a été mis en place et compacté par couches, à une compacité supérieure à $95 \%$ de l'Optimum Proctor Modifié.

Le radier du réacteur constitue une dalle circulaire de $38,80 \mathrm{~m}$ de diamètre. Son épaisseur, en périphérie, au droit de la galerie de précontrainte des câbles, est de $5 \mathrm{~m}$ sur $3 \mathrm{~m}$ de large. Elle passe progressivement à $3,5 \mathrm{~m}$ au centre sur $12 \mathrm{~m}$ (voir figure 3 , REP 900).

Ainsi le radier en béton armé repose sur une couche de sable de remblai compacté dont les caractéristiques moyennes ont été estimées à : $E_{\mathrm{s}}=200 \mathrm{MPa}$ et $v_{\mathrm{s}}=0,25$.

La répartition des contraintes au contact sol-plaque a été étudiée par de nombreux auteurs (BURLAND et al., 1977).

En particulier, BROWN (1969) donne la répartition des contraintes au contact sol-plaque circulaire, supportant une charge verticale centrée et répartie, reposant sur un milieu indéfini, en fonction du coefficient $K$ de rigidité relative :

$$
K=\frac{E_{P}}{E_{S}}\left(1-v_{s}^{2}\right)\left(\frac{t}{R}\right)^{3}
$$

$E_{p}$ : module d'Young du matériau constituant la plaque,

$\mathrm{E}_{\mathrm{S}}, u_{\mathrm{S}}$ : caractéristiques élastiques du sol,

$t, R$ : épaisseur et rayon de la plaque,

$\mathrm{K}=0$ pour une plaque souple,

$\mathrm{K}=\infty$ pour une plaque rigide.

Dans ce dernier cas, la répartition de la pression en fonction de celle moyenne $\mathrm{p}_{\mathrm{m}}$ et de la distance $r$ au centre s'écrit :

$$
p(r)=0,5 P_{m}\left[1-\left(\frac{r}{R}\right)^{2}\right]-\frac{1}{2}
$$

Pour le radier en béton : $E_{p}=20000 \mathrm{MPa}$

$$
\mathrm{R}=19,40 \mathrm{~m}
$$

L'épaisseur est de $3,50 \mathrm{~m}$ au centre et $5 \mathrm{~m}$ en périphérie.

Le coefficient de rigidité, dans ce cas, est inférieur à 0,1 .

Si on se reporte aux courbes données par BROWN (1969) (pour une valeur de $v_{\mathrm{s}}=0,3$ ) la répartition est la suivante: 


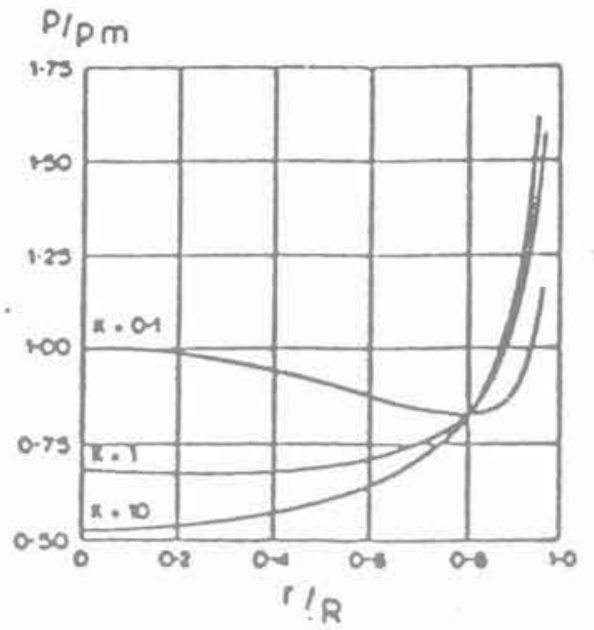

Or, l'instrumentation de la sous-face du radier, avec des cellules GLÖTZL, suivant deux diamètres perpendiculaires, a permis d'obtenir les courbes de répartition de contraintes données ci-après avec deux cas de comportement du radier sous la même charge totale. En effet, en décembre, le puits de cuve reposait au centre de la dalle, sur des appuis en sable, et en janvier, il a été accroché aux planchers supérieurs.

Les courbes montrent le changement de rigidité relative du radier entre décembre 1978 et janvier 1979 du fait de la modification de la répartition de la même charge totale.

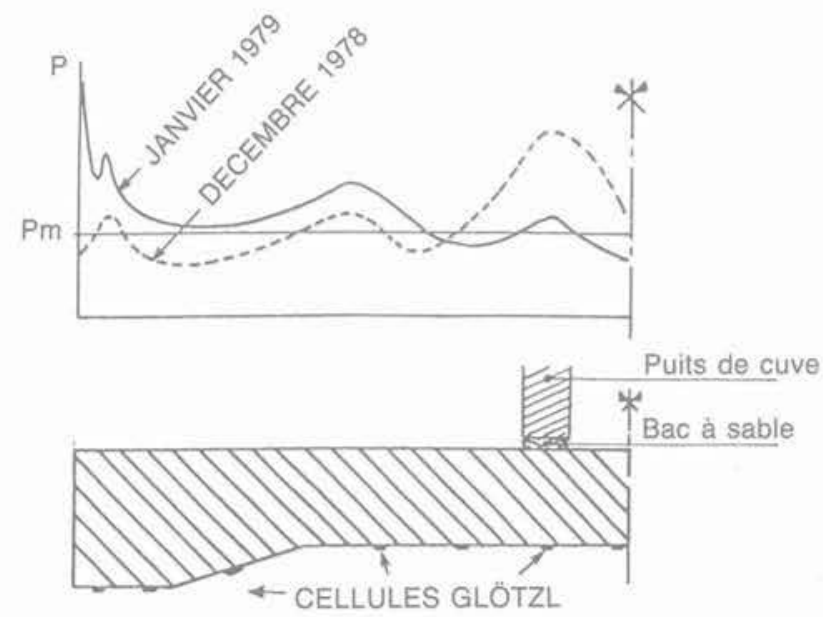

\section{Annexe 3 - Gravelines : Site de la Mer du Nord (REP $900 \mathrm{MWe}$ )}

Ici, le substratum tertiaire constitué de l'Argile Yprésienne d'une puissance de plus de $100 \mathrm{~m}$, est recouvert de plus de $25 \mathrm{~m}$ de dépôts quaternaires comprenant :

- à la base, des sables grossiers avec galets roulés, témoins d'une transgression marine,
- au-dessus, des sables fins, de granulométrie uniforme, avec des passages plus coquilliers, et d'autres comportant des limons organiques, témoins de variations cycliques du niveau moyen des mers.

Des reconnaissances très fines, au pénétromètre statique PAREZ, ont confirmé une certaine homogénéité horizontale des niveaux. L'expérience acquise pour des fondations de charges équivalentes à celle des réacteurs (hauts fourneaux, silos...) avait permis de caractériser les couches par leur module de déformation verticale $E$ à partir de la résistance de pointe au pénétromètre statique. Le calcul des tassements des alluvions quaternaires a été effectué suivant la méthode simplifiée de détermination des contraintes verticales dans chaque couche, par application des coefficients donnés dans les Tables de M. GIROUD (le sol étant considéré comme un milieu linéaire) en tenant compte de la rigidité éventuelle du radier, puis en superposant l'effet, au point considéré, des ouvrages voisins.

Le tassement résultant de l'Argile Yprésienne était obtenu à partir de la carte des contraintes à sa surface. Ainsi, les tassements calculés, à moyen terme, atteignaient-ils :

- 40 à $44 \mathrm{~cm}$ pour le réacteur:

- 20 à $30 \mathrm{~cm}$ pour la majeure partie des ouvrages périphériques de l'îlot nucléaire.

Ces valeurs, importantes à priori, ne faisaient que corroborer celles mesurées pour des ouvrages de charge et emprise analogues à l'usine sidérurgique de Dunkerque, sur un terrain assez semblable.

Comme une réduction sensible de ces tassements n'aurait pu être obtenue que par l'amélioration des caractéristiques d'une couche de limon à $20 \mathrm{~m}$ de profondeur, mais n'aurait pas entraîné de façon assez importante celle des tassements différentiels éventuels entre ouvrages, on a opté pour une autre solution de fondation, originale, mais qui avait déjà été réalisée pour une cokerie et une aciérie. Elle consiste en la réalisation, sous les radiers de béton armé des différents ouvrages (réacteur et îlot nucléaire), d'une dalle constituée d'un mélange de laitier de haut fourneau concassé, provenant de fosse et granulé, ce dernier donnant lieu à une prise hydraulique activée par de la chaux jouant le rôle de catalyseur. Des armatures d'acier de diamètre 32 à $40 \mathrm{~mm}$ y sont incorporées.

Ainsi, la dalle augmente sensiblement la rigidité des radiers et modifie légèrement la répartition des contraintes sur le sol. Mais, surtout, elle assure une progressivité des déformations à la limite des ouvrages contigus de l'îlot nucléaire.

De plus, à la construction, on a prévu une surhauteur de calage de :

- $30 \mathrm{~cm}$ pour le réacteur:

- $20 \mathrm{~cm}$ pour les ouvrages voisins

de façon à éviter d'avoir, du fait des tassements, d'une part un sous-calage général et d'autre part des marches importantes d'un ouvrage à l'autre. 
Le site de Gravelines, dont la construction a débuté en 1973, n'a pas été retenu par E.D.F. pour la mise en place, sous la dalle et les radiers, de cellules de mesure de pression, comme au Blayais. Il a simplement été procédé à des mesures de nivellement.

- Le radier du réacteur a des caractéristiques analogues à celui du Blayais, vu ci-avant.

- La dalle de mélange ternaire a une sous-face horizontale et, de ce fait, une épaisseur de :

- 2,60 m en périphérie,

- $4,10 \mathrm{~m}$ au centre.

L'épaisseur totale du radier et de la dalle est de 7,60 m. Les caractéristiques mécaniques de la dalle de mélange de laitier sont :

- Module de déformation verticale : $\mathrm{E}=10.000 \mathrm{MPa}$

- Coefficient de Poisson : $\nu=0,3$.

En raison, tant de la difficulté d'obtenir les caractéristiques mécaniques des milieux, en tenant compte de leur anisotropie ou non-homogénéité, que de la complication à laquelle pouvaient conduire les calculs, il a été décidé de se ramener à des modèles simples. Ainsi, pour des couches horizontales, supposées chacune homogène et en ne prenant en compte que les contraintes verticales, la méthode de PALMER et BARBER (1940) permet, dans le cas de 2 couches, de déterminer une épaisseur équivalente de la première ayant les caractéristiques de la seconde. DE BARROS (1966) a proposé, dans le cas de 3 couches, une méthode approchée pour obtenir un module de déformation verticale équivalent d'une couche de même épaisseur totale que les 2 premières :

$$
E_{e}=\left[\frac{h_{1} E_{1}-\frac{1}{3}+h_{2} E_{2}-\frac{1}{3}}{h_{1}+h_{2}}\right]^{3}
$$

Ainsi, Tensemble (radier et dalle d'assise en mélange de laitier), aurait un module équivalent de déformation verticale :

- $14000<\mathrm{E}_{e}<16000 \mathrm{MPa}$

- avec un coefficient de Poisson : $0,25<v<0,3$

l'épaisseur de l'ensemble: $h_{1}+h_{2}=7,6 \mathrm{M}$.

A Gravelines, les alluvions entre la dalle de laitier et l'argile, sur près de $20 \mathrm{~m}$ de profondeur, ont un module de déformation verticale, en sollicitation statique, de $25 \mathrm{MPa}$.

Ainsi, la rigidité relative de l'ensemble dalle-radier avec :

$E_{p}=14000 \mathrm{MPa}$

$\mathrm{E}_{\mathrm{S}}=25 \mathrm{MPa}$
$\mathrm{t}=7,60 \mathrm{~m} \quad \mathrm{R}=19,40 \mathrm{~m}$

serait de $\mathrm{K}=30$.

Même pour le tiers de cette valeur, l'ensemble se comporterait, en ce qui concerne la répartition des pres- sions sous le réacteur, d'après les graphiques de BROWN vus ci-avant, comme une fondation très rigide par rapport au sol d'assise. Ceci légitime, dans le cas de Gravelines, pour la détermination des contraintes verticales dans le calcul des tassements aux points considérés, le choix des coefficients d'influence d'une fondation infiniment rigide pour le réacteur.

A partir des tassements en surface du sol, calculés comme il a été indiqué précédemment, en différents points de l'îlot nucléaire, et en tenant compte de ceux induits dans les différentes couches de sol par chacun des ouvrages, il a été possible d'établir une carte prévisionnelle. Celle-ci présentait, tout autour du réacteur, l'aspect d'une "assiette à soupe » un peu irrégulière. La pente de cette "dépression" atteignait une dizaine de centimètres sur quelques mètres. Or, si la dalle de mélange de laitier avec les armatures était assez souple pour épouser les tassements, on pouvait penser que, entre elle et les radiers des bâtiments constituant, à un stade avancé de la construction, des structures rigides, un décollement pouvait se produire en périphérie du réacteur.

Ainsi, on a réservé dans les radiers, au voisinage de quelques repères de nivellement, des orifices permettant de contrôler le niveau de la surface de la dalle de laitier. Leur position est repérée sur le schéma ci-après (figure G1) :

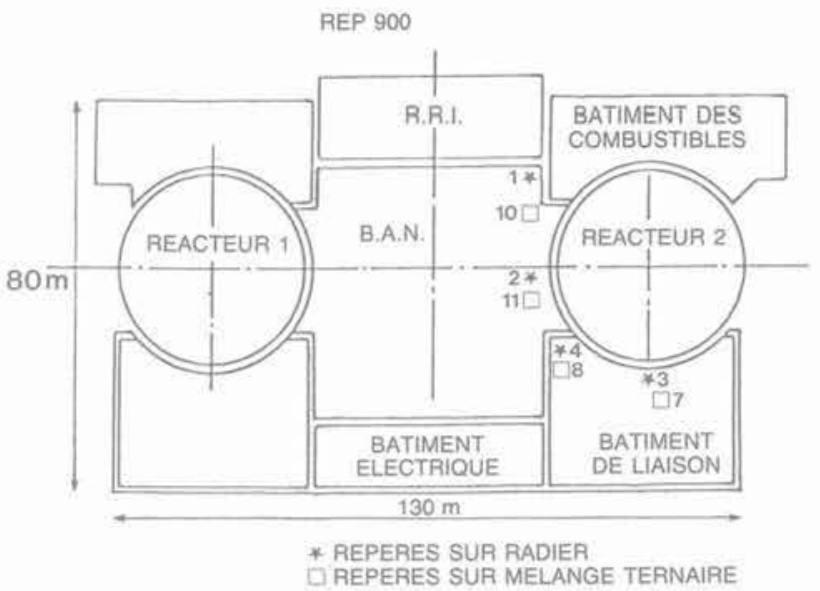

Fig. G1. - Profils géologiques, réacteurs - salles des machines.

Pour les périodes suivantes d'octobre 1975 à janvier et avril 1976, dates auxquelles les charges de génie civil atteignaient près de 60 et $80 \%$ de leur valeur totale, on notait les écarts de tassements suivants :

\begin{tabular}{|c|c|c|}
\hline \multirow{2}{*}{ Couples de repères } & \multicolumn{2}{|c|}{ Écarts (en mm) } \\
& Janvier 1976 & Avril 1976 \\
\hline $1-10$ & 5,4 & 7 \\
$2-11$ & 3,2 & 2 \\
$3-7$ & 11,1 & - \\
$4-8$ & 0,9 & - \\
\hline
\end{tabular}


Malheureusement, la série de mesures sur la dalle a été interrompue. Nénmoins, celles effectuées ont bien confirmé le décollement local, en périphérie du réacteur, entre le radier des ouvrages et la dalle de laitier.

Les mesures de tassements effectuées sur des repères au nombre de 12 placés dans la galerie de précontrainte périphérique du réacteur, à l'intérieur du radier, ont bien montré, au fur et à mesure de la construction de l'enceinte, que le radier, en périphérie, se comportait comme un anneau de plus en plus rigide.

\section{Annexe 4 - Cas de Nogent-sur-Seine (REP $1300 \mathrm{MWe}$ )}

La centrale nucléaire de Nogent-sur-Seine est implantée en rive droite de la seine à $2,5 \mathrm{~km}$ au nord-est de Nogent-sur-Seine, $100 \mathrm{~km}$ à l'amont de Paris. Le projet dans sa phase actuelle, comporte la construction de deux tranches de 1300 MWe (palier P'4).

D'une façon générale, la géologie du site est constituée par une couche d'alluvions sablo-graveleuses qui surmonte un substratum crayeux sub-horizontal, comportant une zone altérée en sa partie haute (figure N1).

\section{Campagnes de reconnaissance}

Un première campagne de reconnaissance succincte a été menée en 1976 pour établir l'avant projet et les pre- mières décisions sur le choix du type de fondation. Elle a été suivie par deux autres campagnes beaucoup plus vastes en 1978 et 1979 comportant 156 points de reconnaissance, recouvrant une superficie de 200 ha. Elles comportaient (figure N2) :

- 24 sondages carottés

- 37 forages pressiométriques type MENARD

- 14 pénétromètres statiques type PAREZ

- 78 pénétromètres dynamiques type SERMES

- 5 forages destructifs avec enregistrement de paramètres, type LUTZ

- 3 puits de gros diamètre.

256 échantillons, prélevés dans des sondages carottés ou des puits, ont été testés au laboratoire.

Malgré le nombre assez élevé de points de reconnaissance, il y a eu donc en moyenne moins de 1 point de reconnaissance par hectare pour l'ensemble du site. La zone d'implantation des îlots nucléaires comportait une densité plus élevée de points de reconnaissance (1 pour $1000 \mathrm{~m}^{2}$ ), ce qui peut paraitre encore insuffisant (SIGISMOND et al., 1983). Mais il faut rappeler que, sur un site prévu pour l'implantation d'une centrale nucléaire, les campagnes de reconnaissance doivent être menées dans un laps de temps réduit entre la possibilité d'accès sur le site et le début des travaux proprement dits.
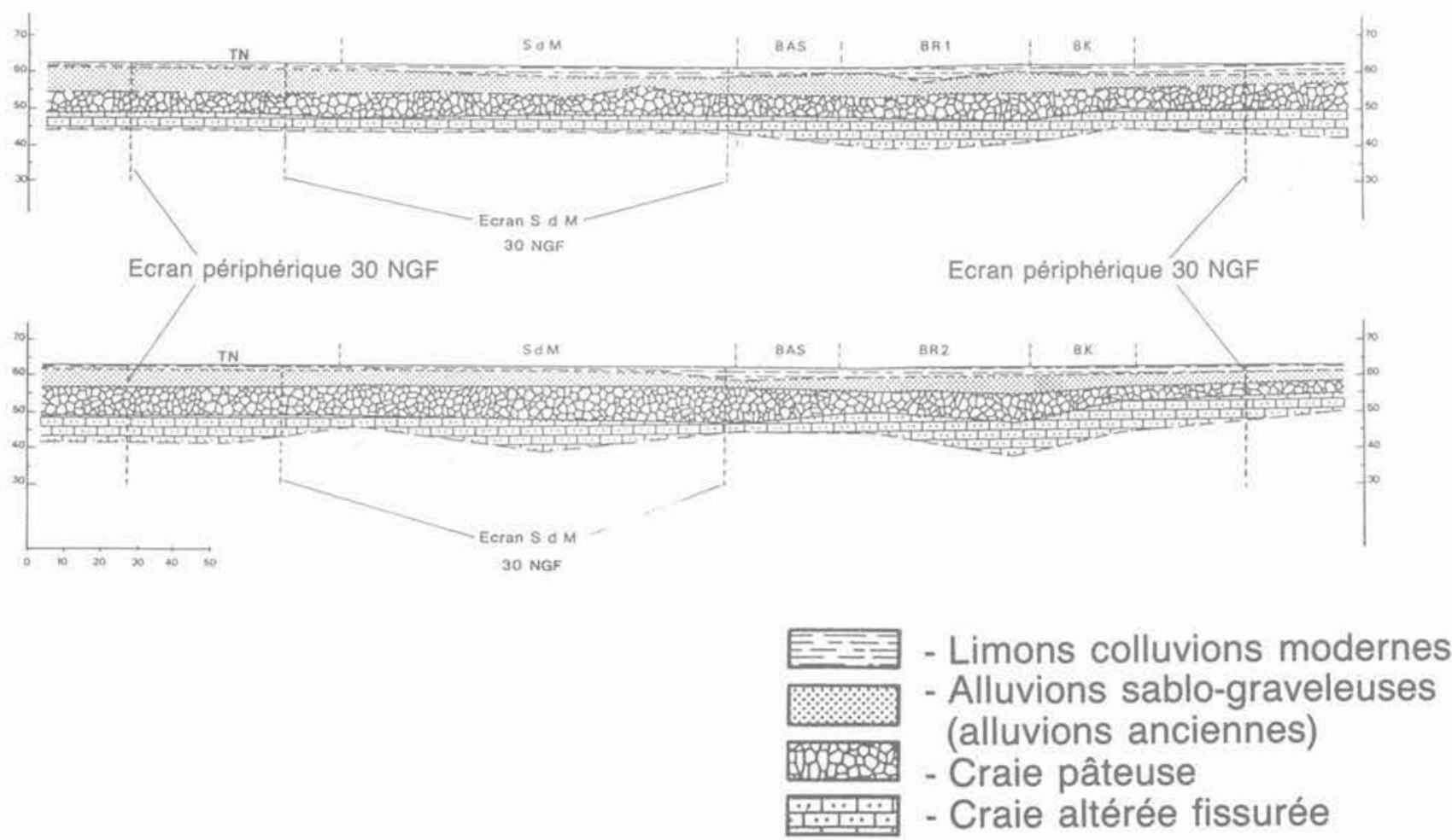

Fig. N1. - Nogent-sur-Seine - Profils du sol.

Fig. N1. - Soil profils. 


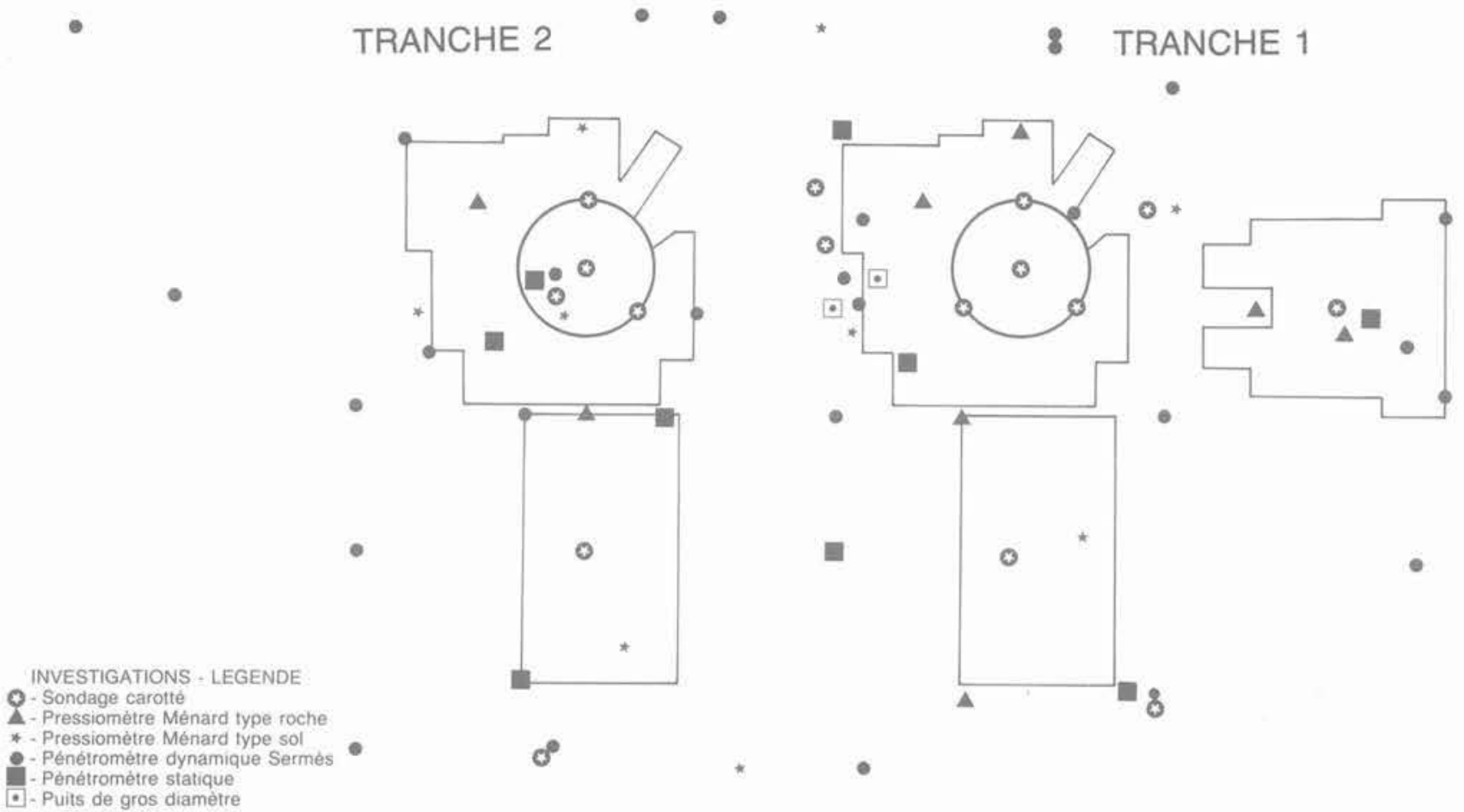

Fig. N2. - Nogent-sur-Seine - Schéma d'implantation des reconnaissances (îlots nucléaires).

Fig. N2. - Site investigation (nuclear is/and).

\section{La stratigraphie}

L'analyse des reconnaissances et essais réalisés dans la zone d'implantation de la centrale a conduit à distinguer plusieurs couches successives à partir du terrain naturel, établi à une cote pratiquement constante de $63 \mathrm{NGF}$ :

- les limons (1 à 3 mètres) ;

- des alluvions sablo-graveleuses, d'épaisseur variant de 4 à $6 \mathrm{~m}$ (moyenne $4 \mathrm{~m}$ );

- le substratum crayeux, dont la partie haute est très fortement altérée, a été subdivisé en trois différents faciès :

- «la craie pâteuse», qui est composée de nodules centrimétriques de craie dure enrobés dans une matrice pâteuse née de l'altération de la craie rocheuse. L'épaisseur de cette couche est extrêmement variable ( 4 à $12 \mathrm{~m}$ ), ce qui confère à sa base un profil très accidenté

- la craie altérée fissurée (ou de transition), ensemble très fragmenté de morceaux de craie de dimensions diverses sur 4 à $8 \mathrm{~m}$ d'épaisseur ;

- la craie rocheuse, localement fracturée, établie sur plusieurs centaines de mètres, dont le toit se situe entre 20 et $25 \mathrm{~m}$ de profondeur.

La couche de la craie pâteuse se caractérise par son hétérogénéité et la médiocrité générale de ses propriétés géotechniques. Les premiers mètres de la craie pâteuse, au contact des alluvions, présentent les caractéristiques mécaniques les plus faibles. Au pressiomètre la valeur de la pression limite de la craie pâteuse est en moyenne de $0,8 \mathrm{MPa}$, mais peut localement descendre à $0,5 \mathrm{MPa}$ (figure $\mathrm{N} 3$ ) ; son module pressiométrique varie de 4 à $20 \mathrm{MPa}$ (valeur moyenne $8 \mathrm{MPa}$ ). Le module œedométrique mesuré au laboratoire, est de l'ordre de $21 \mathrm{MPa}$.

\section{Principe de fondation}

Les caractéristiques géotechniques de la craie pâteuse étant trop faibles pour recevoir directement les fondations superficielles des îlos nucléaires, diverses solutions permettant de la solliciter à des contraintes acceptables ont été étudiées dont la substitution de la craie pâteuse, le préchargement par rabattement et remblai, la fondation sur pieux.

a. PENETROMETRES DYNAMIQUES

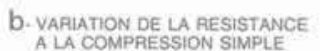
A LA COMPRESSION SIMPLE
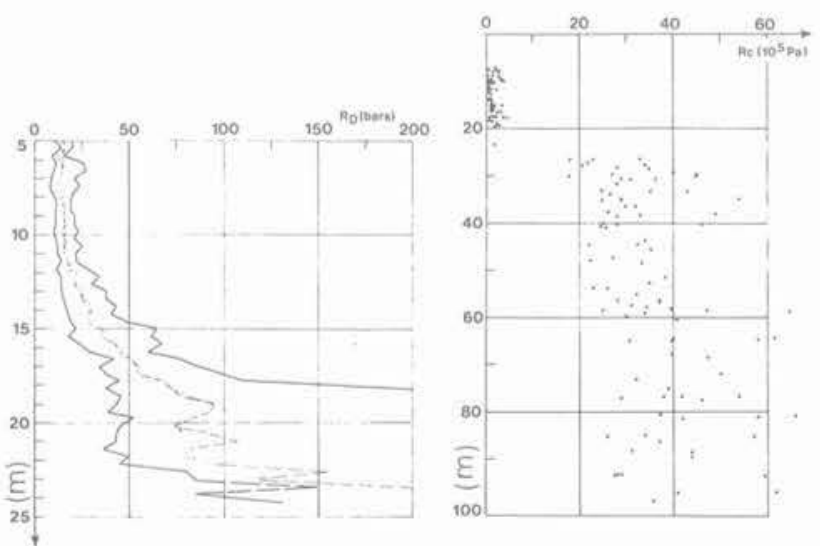
C. ESSAIS PRESSIOMETAIQUES d- ESSAIS PAESSIOMETRIOUES CAMAE PATEUSE CAAIE PATEUSE
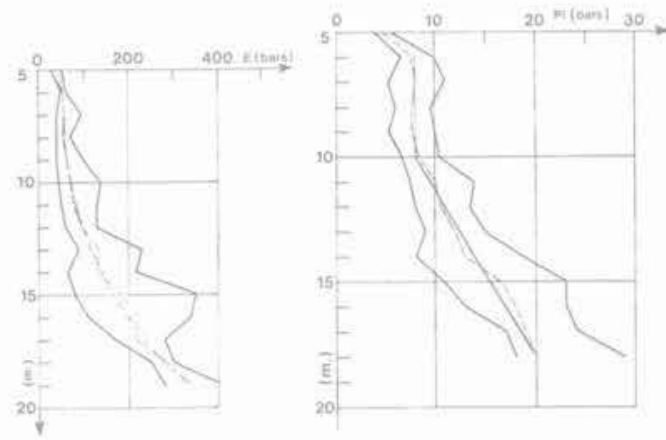

Fig. N3. - Nogent-sur-Seine - Les résultats des essais de compression simple (b), pénétromètres dynamiques (a), pressiomètres (c et d).

Fig. N3. - Some of the Tests Results.
La solution retenue pour les îlots nucléaires consiste en la réalisation d'une plate-forme calée à la cote 68.20 NGF sur laquelle sont implantées les deux tranches et la station de pompage. Les fondations des principaux bâtiments sont situées en majeure partie à 60,50 NGF (soit environ $4 \mathrm{~m}$ au-dessus du toit de la craie pâteuse). Elles sont réalisées à sec par rabattement de la nappe et terrassement à l'intérieur d'un dispositif d'étanchéité comprenant des enceintes étanches ancrées dans la craie rocheuse à la cote 28 NGF

\section{Prévisions des tassements}

Les premières estimations de tassements, réalisées par l'ingénieur-conseil en 1979. étaient basées sur les résultats des essais cedométriques de la campagne 1978, avec deux séries d'hypothèses :

a) déformations œdométriques (calcul œdométrique classique) :

b) calcul élastique $3 \mathrm{D}$, avec un module d'Young déduit de module œedométrique.

Ces calculs prévoyaient un tassement moyen d'environ $15 \mathrm{~cm}$ pour le bâtiment du réacteur en fin de construc-

CHARGES (Tonne)

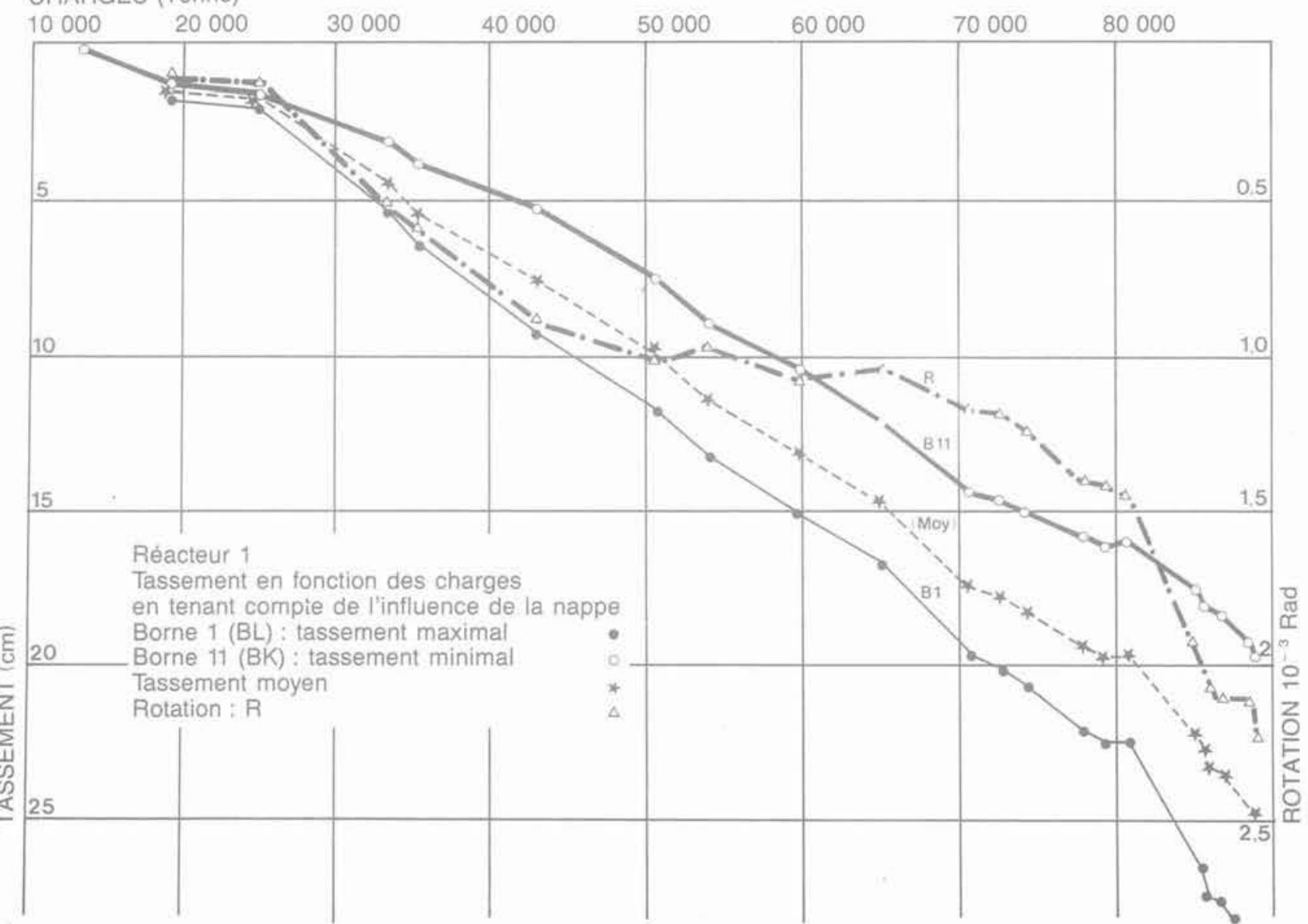

Fig. N4. - Nogent-sur-Seine - Suivi des tassements du réacteur 1 (auscultation altimétrique) Fig. N4. - Measured settlements of Reactor Building 1. 


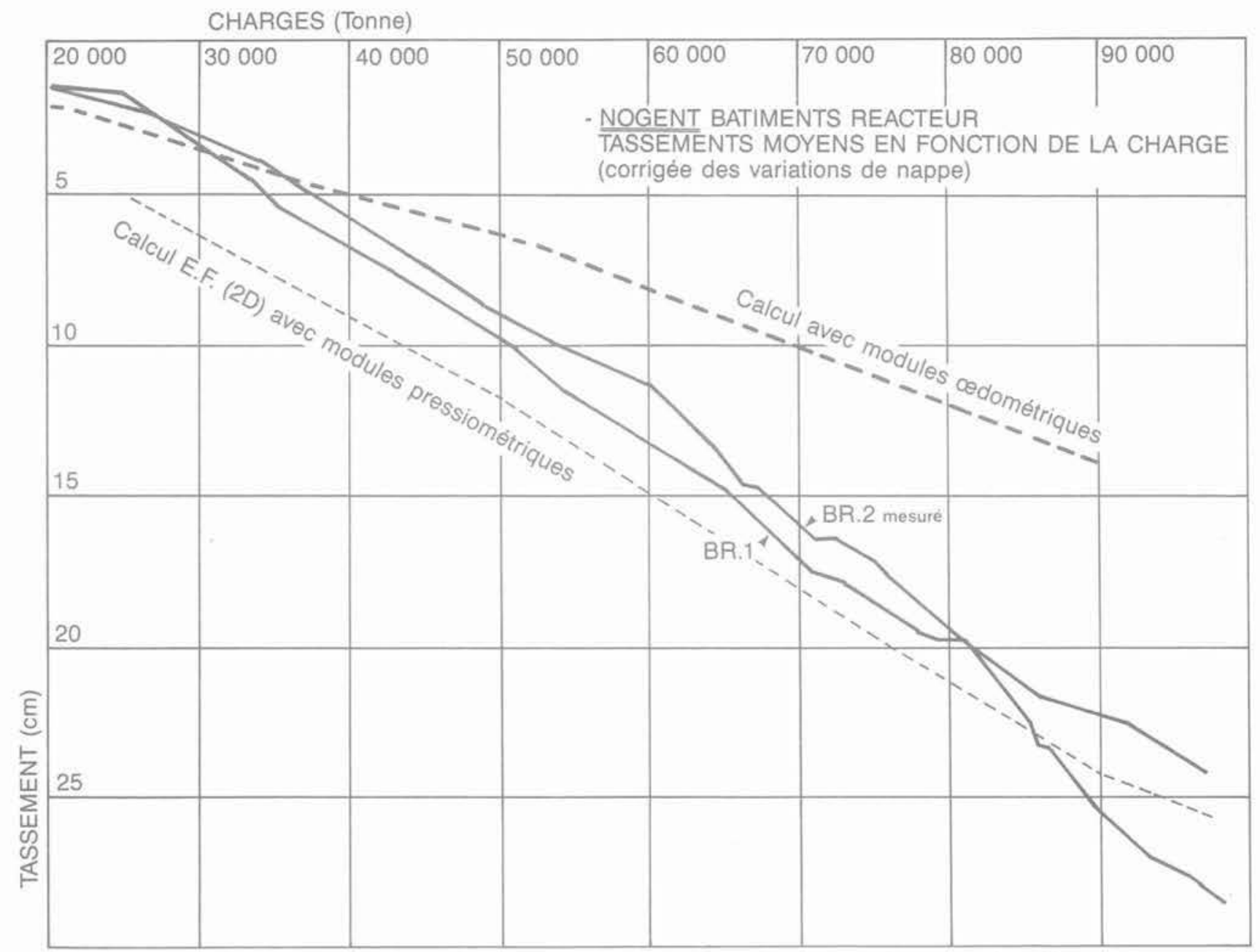

Fig. N5. - Comparaison des tassements moyens des réacteurs 1 et 2 avec les valeurs calculées.

Fig. N5. - Comparison of measured and calculated settlements of Reactor Buildings.

tion (contrainte moyenne de $50 \mathrm{t} / \mathrm{m}^{2}$ ), et un léger dévers de celui-ci, de l'ordre de $1.10^{-4}$, orienté vers le bâtiment combustible (BK), appliquant une contrainte movenne de $36 \mathrm{t} / \mathrm{m}^{2}$ au sol. L'hétérogénéité de la craie pâteuse et en particulier les variations d'épaisseur. avaient conduit à envisager différentes hypothèses de calcul prévoyant la possibilité de dévers plus importants, sans qu'il soit possible cependant de les chiffrer d'une façon précise.

L'estimation des tassements basée sur une approche pressiométrique avait conduit à des valeurs sensiblement plus êlevées (tassement moyen du bâtiment réacteur supérieur à $30 \mathrm{~cm}$ ).

Une autre série de calculs de tassements a été aussi effectuée en se basant principalement sur les résultats des essais triaxiaux, et en assimilant les courbes effortdéformation à des hyperboles (loi de DUNCAN). La distinction est faite aussi entre la partie sphérique et déviatorique du tenseur des déformations. Ces derniers calculs, revus en 1982, prévoyaient un tassement moyen de l'ordre de $20 \mathrm{~cm}$ pour le bâtiment réacteur à sa charge finale.

\section{Confrontation prévisions - observations}

D'après les mesures réalisées sur les tassomètres, mis en place sous les remblais de préchargement des réfrigérants atmosphériques et à côté des bâtiments du réacteur, l'essentiel des tassements observés (environ $80 \%$ ) se situe dans lhorizon de la craie pâteuse (DAVESNE et al., 1983).

La construction du bâtiment réacteur de la tranche 1 a été commencée en août 1981. Des écarts notables entre les prévisions basées sur les modules œdométriques et les observations sont remarqués dès 1982 . quand la charge n'était qu'à 50 \% de sa charge finale (figure N5).

En auril 1984, le bâtiment réacteur 1 était à $80 \%$ de sa charge finale, son tassement moyen avait atteint $20 \mathrm{~cm}$ et son basculement était de l'ordre de 1,5 × 10-3 radiant (figure N4). Celui-ci était orienté vers le bâtiment électrique (BAS-BL: $23 \mathrm{t} / \mathrm{m}^{2}$ ) plus léger que le bâtiment combustible (BK; $\left.36 \mathrm{t} / \mathrm{m}^{2}\right)$. Par contre, le dévers du bâtiment réacteur 2 a été beaucoup plus faible et ceux des autres bâtiments de cette tranche ont suivi une allure plus conforme aux prévisions (figure N6). 


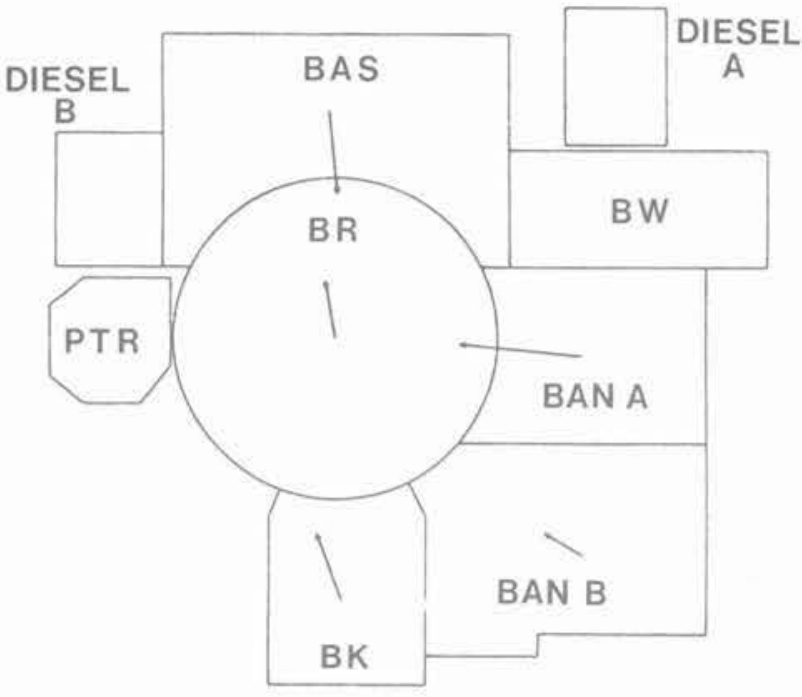

$\longmapsto 2 \mathrm{~mm} / \mathrm{m}$

TRANCHE 1 - Charge (réacteur) : 91400 T

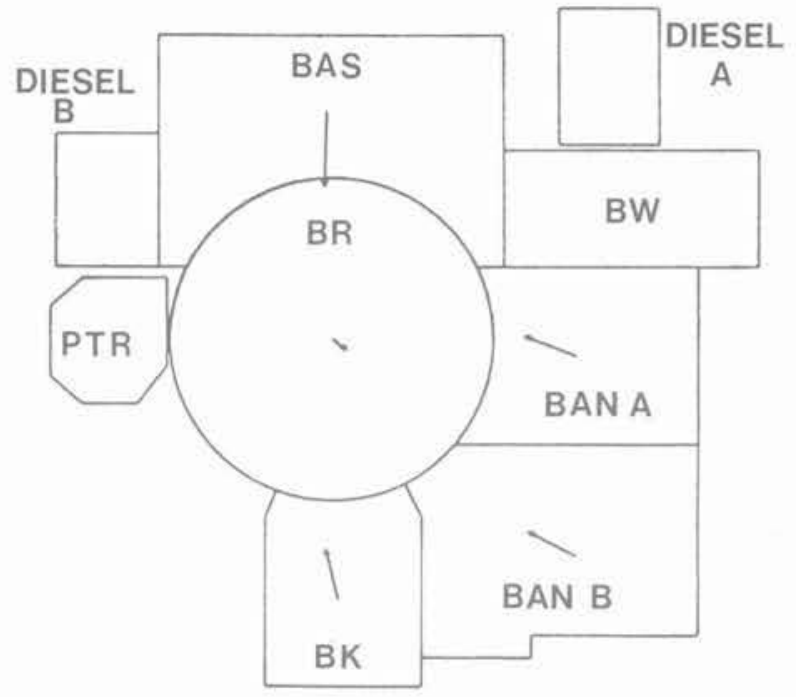

$-2 \mathrm{~mm} / \mathrm{m}$

TRANCHE 2 - Charge (réacteur) : 92700 T

Fig. N6. - Nogent-sur-Seine - Basculement des radiers.

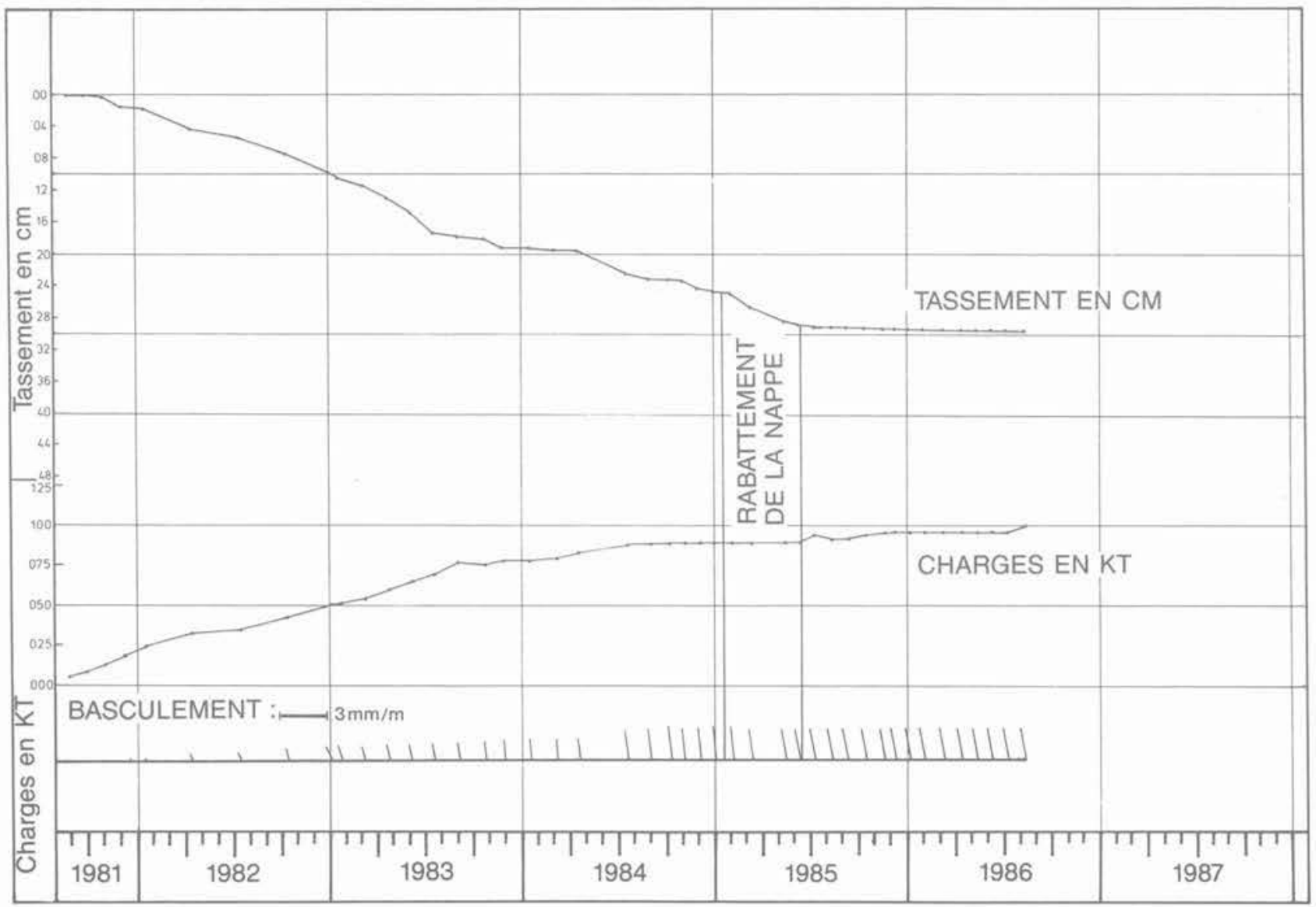

Fig. N7. - Reacteur 1 de Nogent-sur-Seine. Tassement et basculement du centre du radier. Fig. N7. - Settlement and Tipping of the center of the Raft. 
Pour éviter une accentuation des dévers des bâtiments après la pose des installations, il a été décidé d'effectuer une opération de préchargement (sur les tranches 1 et 2). Il s'agissait de soumettre l'ensemble des bâtiments à leurs charges maximales avant la mise en place des matériels électromécaniques. Ce préchargement exécuté entre le 28 janvier 1985 et le 15 juin 1985 comporte :

- le rabattement de la nappe phréatique de la cote 61 NGF à 56 NGF ;

- le chargement par pondéreux du BR1 (1 570 t) et du BK1 (2000 t) ;

- l'anticipation de l'épreuve enceinte.

En juillet 1985 la nappe phréatique est relâchée au fur et à mesure que l'on introduit le matériel dans le BR1. Les charges actives apportées sont sensées équilibrer la diminution des contraintes dans la craie pâteuse due à la remontée du niveau piézométrique.

Cette opération a conduit, comme prévu, à une stabilisation de tassement et basculement du bâtiment réacteur 1 (figure N7).

Parallèlement une nouvelle campagne de reconnais sance et des études ont été engagées pour rechercher les causes du dévers du bâtiment réacteur 1 et de la sous-estimation des tassements moyens.

Diverses hypothèses ont été étudiées comme :

- variation de l'épaisseur des couches du sol;

- rupture locale par cisaillement de la couche des alluvions et entrée en plasticité de la couche supérieure de la craie pâteuse;

- influence de l'histoire réelle des chargements et de leur excentrement.
Plusieurs calculs aux éléments finis ont été effectués avec diverses lois de comportement: élastique linéaire, modèle multilinéaire, modèle élastoplastique parfait, et modèle élastoplastique multimécanismes.

Le dernier calcul, mené comme s'il s'agissait d'un calcul d'avant projet basé sur des paramètres déduits des essais de laboratoire, a conduit aussi à une sousestimation des tassements observés. Il faut noter également que les nombreux paramètres de la loi ne pouvaient pas être évalués avec suffisamment de précision à partir des résultats expérimentaux alors disponibles.

Les calculs paramétriques aux E.F. 2D (figure N8), exécutés avec la loi multilinéaire et des modules du sol déduits des essais pressiométriques ont conduit à des tassements moyens proches de ceux observés sur site (figure N5). Ils ont montré aussi que le dévers du bâtiment réacteur peut s'expliquer par l'existence d'une zone plus faible, au sein de la couche de la craie pâteuse, côté bâtiment électrique.

La modélisation des courbes pressiométriques par utilisation des paramètres issus des essais de laboratoire a montré que ceux-ci conduisent généralement à une surestimation assez importante des caractéristiques mécaniques de la craie pâteuse. Les causes peuvent être recherchées en une grande sensibilité de la craie pâteuse aux remaniements et à la texture particulière de celle-ci (en particulier l'existence des nodules).

Des études supplémentaires sont actuellement en cours pour mieux cerner le comportement in situ et au laboratoire de la craie pâteuse. Elles seront suivies pour une nouvelle modélisation aux E.F. du cas Nogent, dans le cadre d'une étude plus vaste entreprise par E.D.F.-SEPTEN pour la révision de différentes méthodes de calcul des tassements.

\section{RADIER BAS}

RADIER BR

RADIER BK

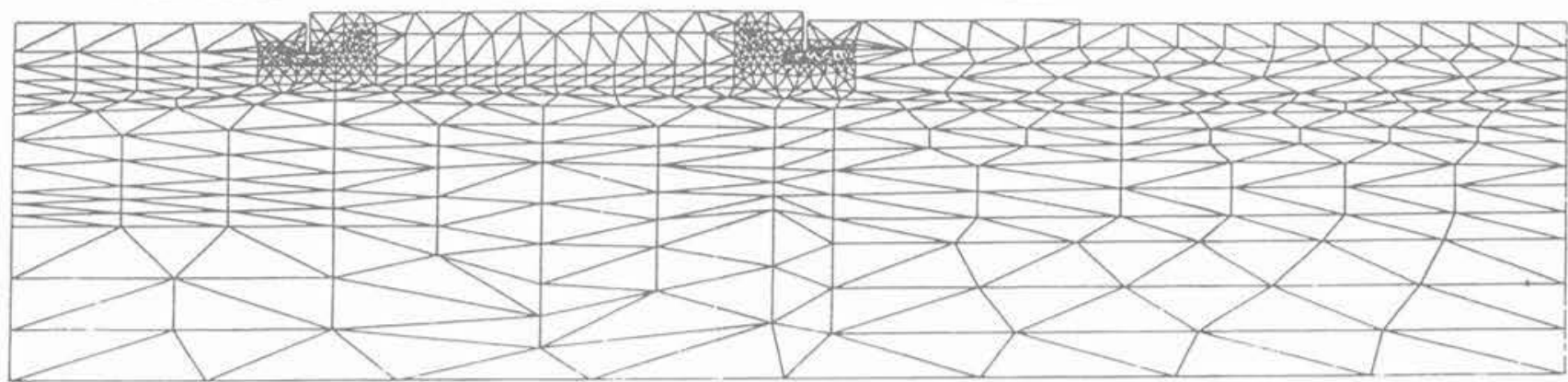

Fig. N8 - Nogent-sur-Seine - Maillage E.F. $2 D$ (utilisé pour l'étude paramétrique). Fig. N8 - F.E. Mesh (2D) used for parametric study. 


\section{E.N.P.C./D.F.C.A.I. - SESSIONS DE FORMATION - $1^{\text {er }}$ SEMESTRE 1988}

\section{géotechnique, matériaux, structures}

Journées d'étude :

- Grands travaux et géologie

- La responsabilité du géotechnicien : pratique professionnelle et aspects jurisprudentiels

- Le creusement mécanisé des tunnels

- L'évaluation des risques naturels

- La pratique de l'injection et des écrans d'étanchéité

- Stockage en souterrain

- Stabilité des pentes

- Les nouvelles règles parasismiques

- Géotechnique : les enseignements à tirer des accidents survenus aux ouvrages de génie civil

\begin{tabular}{|c|c|}
\hline $\begin{array}{l}18 \text { et } 19 \text { novembre } \\
15 \text { et } 16 \text { décembre }\end{array}$ & $\begin{array}{l}\text { Paris } \\
\text { Paris }\end{array}$ \\
\hline $\begin{array}{l}15 \text { au } 17 \text { décembre } \\
1 \text { er et } 2 \text { mars } \\
8 \text { au } 10 \text { mars } \\
22 \text { au } 24 \text { mars } \\
26 \text { au } 29 \text { avril } \\
1 \text { er et } 2 \text { juin } \\
\text { Journées d'étude }\end{array}$ & $\begin{array}{l}\text { Paris } \\
\text { Paris } \\
\text { Paris } \\
\text { Paris } \\
\text { Grenoble } \\
\text { Grenoble }\end{array}$ \\
\hline
\end{tabular}

Pour toute information, s'adresser à l'E.N.P.C., 28, rue des Saints-Pères, 75007 Paris à Christine ROSE. Tél. 16 (1) 42.60.34.13,

\section{CENTRIFUGEUSE 88}

Congrès international sur la modélisation géotechnique en centrifugeuse.

Paris, 25-27 avril 1988.

Sous l'égide de la Société Internationale de Mécanique des Sols et de Travaux de Fondations, sous le patronage du Comité Français de Mécanique des sols et des Fondations.

Thèmes du congrès :

- Applications de la modélisation en centrifugeuse à la conception et au dimensionnement des ouvrages : fondations, ouvrages en terre, ouvrages de soutènement, tunnels, etc.

- Centrifugeuses et instrumentation : présentation d'installations, conception des centrifugeuses, présentation de moyens de mesure, d'équipements, etc.

- Problèmes dynamiques : chocs, explosions, simulation de séismes, etc.

- Évaluation des résultats d'essais en centrifugeuse : confrontation de résultats d'essais sur modèle avec essais en vraie grandeur, essais croisés, analyse des questions de similitude, etc.

- Nouvelles frontières pour la modélisation en centrifugeuse : problèmes de mécanique des fluides dans les sciences de la terre, mécanique de la glace, tectonique, écoulements de pondéreux en silos, etc.

Renseignements : M. J.F. CORTE - Laboratoire Central des Ponts-et-Chaussées, B.P. 19, 44340 BOUGUENAIS (France). Tél. 40,32.06.66. 